\title{
Three-body nuclear forces from a matrix model
}

\author{
Koji Hashimoto $^{a}$ and Norihiro lizuka ${ }^{b}$ \\ ${ }^{a}$ Mathematical Physics Lab., RIKEN Nishina Center, \\ Saitama 351-0198, Japan \\ ${ }^{b}$ Theory Division, CERN, \\ CH-1211 Geneva 23, Switzerland \\ E-mail: koji@riken.jp, norihiro.iizuka@cern.ch
}

ABstRACT: We compute three-body nuclear forces at short distances by using the nuclear matrix model of holographic QCD proposed in our previous paper with P. Yi. We find that the three-body forces at short distances are repulsive for (a) aligned three neutrons with averaged spins, and (b) aligned proton-proton-neutron/proton-neutron-neutron. These indicate that in dense states of neutrons such as cores of neutron stars, or in Helium$3 /$ tritium nucleus, the repulsive forces are larger than the ones estimated from two-body forces only.

KEYWORDS: Gauge-gravity correspondence, QCD, 1/N Expansion

ARXiv EPRINT: 1005.4412 


\section{Contents}

1 Introduction 1

2 Review: a matrix model and two-body interactions $\quad 2$

2.1 A matrix model action 2

2.2 Two-body nuclear forces 3

3 Three-body baryon interaction $\quad \mathbf{5}$

3.1 An exercise: classical treatment of spin/isospin 6

$\begin{array}{ll}3.2 \text { Generic three-body interactions: a set-up } & 10\end{array}$

3.3 Three-body Hamiltonian for baryons aligned on a line 13

$\begin{array}{lll}4 & \text { Summary and discussions } & 20\end{array}$

\section{Introduction}

One of the fundamental ingredients of nuclear physics is the nuclear force with which pointlike nucleons interact with each other. A variety of aspects of nuclear forces results in the protean metamorphosis of nuclei, the bound states of nucleons. It is known that in nuclear forces there are forces that can not be explained by two-body forces only, one of which is the three-body force. The three-body forces play important role, for example, in reproducing excitation spectra of light nuclei, or explaining equations of states for high-density baryon matters such as supernovae and neutron stars. However, in spite of the long history of nuclear physics, the bulk properties of three-body nuclear forces are yet to be revealed.

The main obstacle for revealing the various aspects of nuclear forces is obvious: QCD is strongly coupled and thus difficult to solve. In this paper, by using a nuclear matrix model of holographic QCD which we have derived together with P. Yi in [1], we explicitly compute a three-body nuclear force in a large $N_{c}$ holographic QCD. The two-body nuclear force was already computed in [1].

For the derivation of our matrix model [1] we use the gauge/string duality (the AdS/CFT correspondence) [2-4] applied to a D4-D8 system [5,6] of a large $N_{c}$ QCD at a large 'tHooft coupling $\lambda$. Precisely speaking, our matrix model is a low-energy effective field theory on baryon vertex D4-branes [7] in the D4-D8 holographic model [5, 6] of large $N_{c}$ QCD. The matrix model describes $k$-body baryon systems with arbitrary $k$, where the size of the matrix is given by this $k$, based on the fact that baryons are wrapped D-branes on sphere [7] in the gravity side of the gauge/string duality. In the previous work [1], in addition to the derivation of the matrix model, the cases with $k=1$ (baryon spectrum) and $k=2$ (two-body nuclear force) were studied. For $k=2$, it was found that a universal repulsive core exists for any baryon states with two flavors. Since our matrix 
model is not a phenomenological model for multi-baryon systems, but based on a firm ground of the gauge/string duality in string theory, it is natural to extend the analysis of our matrix model to derive the three-body nuclear forces. In this paper, we continue the analysis to the $k=3$ case, i.e. we study the short-range three-body nuclear force, using the matrix model.

Although generic configurations of three baryons can be treated in the matrix model, as the computations are involved and thus not so illuminating, in this paper we shall concentrate on two particular examples: (a) three neutrons with spins averaged and (b) proton-proton-neutron (and proton-neutron-neutron), both aligned on a line with equal spacings. System with spin averaged is rather typical for dense states of multi-baryons such as cores of neutron stars. The latter is related to Helium-3 and tritium nucleus. For both cases, the resultant three-body potential is positive, i.e. repulsive. It scales as $N_{c} / \lambda^{2} r^{4}$ (where $r$ is the inter-nucleon distance), in contrast to the two-body repulsive core $\sim N_{c} / \lambda r^{2}$. As the region of validity is at short range, $1 / \sqrt{\lambda} M_{\mathrm{KK}} \ll r \ll 1 / M_{\mathrm{KK}}$ (where $M_{\mathrm{KK}} \sim \mathcal{O}(1 \mathrm{GeV})$ ), the three-body potential is suppressed compared to the twobody potential by $\sim 1 / \lambda\left(r M_{K K}\right)^{2} \ll 1$. However at very short distances, i.e. at high dense states of nucleons, three-body forces are not small.

The organization of this paper is as follows. We first review the matrix model and the two-body calculation shown in [1]. Then in section 3 , we calculate the three-body forces. First, as an exercise, we treat the case with spin/isospins aligned classically, and find that the three-body force vanishes for this case, which is consistent with the soliton approach [8]. After that, we proceed to generic three-body forces with quantum spin/isospins. The final section is devoted for discussions.

\section{Review: a matrix model and two-body interactions}

The procedures of the computation of the three-body nuclear forces is quite analogous to the two-body case performed in [1]. Here we provide a summary of the matrix model action and the computation of the two-body nuclear forces of [1].

\subsection{A matrix model action}

In [1], we proposed with $\mathrm{P}$. Yi a $\mathrm{U}(k)$ matrix model which describes generic $k$-body interaction of nucleons. Note that the rank of gauge group $\mathrm{U}(k)$ is not at all related to the number of colors $N_{c}$ but just the number of nucleons $k$. The matrix model action is quite simple,

$$
\begin{aligned}
S= & \frac{\lambda N_{c} M_{\mathrm{KK}}}{54 \pi} \int d t \operatorname{tr}_{k}\left[\left(D_{0} X^{M}\right)^{2}-\frac{2}{3} M_{\mathrm{KK}}^{2}\left(X^{4}\right)^{2}+D_{0} \bar{w}_{i}^{\dot{\alpha}} D_{0} w_{\dot{\alpha} i}-\frac{1}{6} M_{\mathrm{KK}}^{2} \bar{w}_{i}^{\dot{\alpha}} w_{\dot{\alpha} i}\right. \\
& \left.+\frac{3^{6} \pi^{2}}{4 \lambda^{2} M_{\mathrm{KK}}^{4}}(\vec{D})^{2}+\vec{D} \cdot \vec{\tau}_{\dot{\beta}}^{\dot{\alpha}} \bar{X}^{\dot{\beta} \alpha} X_{\alpha \dot{\alpha}}+\vec{D} \cdot \vec{\tau}_{\dot{\beta}}^{\dot{\alpha}} \bar{w}_{i}^{\dot{\beta}} w_{\dot{\alpha} i}\right]+N_{c} \int d t \operatorname{tr}_{k} A_{0} .
\end{aligned}
$$

The peculiar property of this matrix model is the simplicity: changing the number of the nucleons $k$ is available just by choosing $\mathrm{U}(k)$ for the gauge group of the matrix model. 


\begin{tabular}{|c|c|c|c|c|}
\hline field & index & $\mathrm{U}(k)$ & $\mathrm{SU}\left(N_{f}\right)$ & $\mathrm{SU}(2) \times \mathrm{SU}(2)$ \\
\hline$X^{M}(t)$ & $M=1,2,3,4$ & $\mathrm{adj}$. & $\mathbf{1}$ & $(\mathbf{2}, \mathbf{2})$ \\
\hline$w_{\dot{\alpha} i}(t)$ & $\dot{\alpha}=1,2 ; i=1, \cdots, N_{f}$ & $\mathbf{k}$ & $\mathbf{N}_{\mathbf{f}}$ & $(\mathbf{1}, \mathbf{2})$ \\
\hline$A_{0}(t)$ & & $\mathrm{adj}$. & $\mathbf{1}$ & $(\mathbf{1}, \mathbf{1})$ \\
\hline$D_{s}(t)$ & $s=1,2,3$ & $\mathrm{adj}$. & $\mathbf{1}$ & $(\mathbf{1}, \mathbf{3})$ \\
\hline
\end{tabular}

Table 1. Fields in the matrix model.

In [1] it was demonstrated how to compute the baryon spectrum $(k=1)$ and two-body nuclear forces $(k=2)$ at short distances.

To be concise, here we briefly describe the matter content of the matrix model (2.1). The model has a unique scale $M_{\mathrm{KK}}$, and $\lambda=N_{c} g_{\mathrm{QCD}}^{2}$ is the 'tHooft coupling constant of QCD, with the number of colors $N_{c}$. The field content is summarized in table 1. The dynamical fields are only $X^{M}$ and $w_{\dot{\alpha} i}$, while $A_{0}$ and $D_{s}$ are auxiliary fields. In writing these fields, the indices for the gauge group $\mathrm{U}(k)$ are implicit. In this paper we consider only the two-flavor case $N_{f}=2$ for simplicity. The symmetry of this matrix quantum mechanics is $\mathrm{U}(k)_{\text {local }} \times \mathrm{SU}\left(N_{f}\right) \times \mathrm{SO}(3)$ where the last factor $\mathrm{SO}(3)$ is the spatial rotation, which, together with a holographic dimension, forms a broken $\mathrm{SO}(4) \simeq \mathrm{SU}(2) \times \mathrm{SU}(2)$ shown in the table. The breaking is due to the mass terms for $X^{4}$ and $w_{\dot{\alpha} i}$. In the action, the trace is over these $\mathrm{U}(k)$ indices, and the definition of the covariant derivatives is $D_{0} X^{M} \equiv \partial_{0} X^{M}-i\left[A_{0}, X^{M}\right], D_{0} w \equiv \partial_{0} w-i w A_{0}$ and $D_{0} \bar{w} \equiv \partial_{0} \bar{w}+i A_{0} \bar{w}$. The spinor indices of $X$ are defined as $X_{\alpha \dot{\alpha}} \equiv X^{M}\left(\sigma_{M}\right)_{\alpha \dot{\alpha}}$ and $\bar{X}^{\dot{\alpha} \alpha} \equiv X^{M}\left(\bar{\sigma}_{M}\right)^{\dot{\alpha} \alpha}$ where $\sigma_{M}=(i \vec{\tau}, 1)$ and $\bar{\sigma}_{M}=(-i \vec{\tau}, 1)$, with Pauli matrices $\tau$. All of these definitions follow the notation of [9]. For the derivation of this matrix model via gauge/string duality, see [1].

\subsection{Two-body nuclear forces}

We review briefly [1] for explaining how to obtain the two-body nuclear forces. First, we describe a single baryon wave function, and second, obtain the two-body Hamiltonian by integrating out $A_{0}$ with a simple gauge choice.

In all the cases, we need to solve the "ADHM constraint" [10] which minimizes the potential induced by integrating out $D_{s}$. This is because the potential has a coefficient $\lambda^{2}$ which is very large in the gauge/string duality.

$$
\vec{\tau}_{\dot{\beta}}^{\dot{\alpha}}\left(\bar{X}^{\dot{\beta} \alpha} X_{\alpha \dot{\alpha}}+\bar{w}_{i}^{\dot{\beta}} w_{\dot{\alpha} i}\right)_{B A}=0
$$

Here $A, B=1, \ldots, k$.

For a single baryon $k=1$, this equation is simply solved by $w_{\dot{\alpha} i}=U_{\dot{\alpha} i} \rho$ where $U$ is an $\mathrm{SU}(2)$ matrix and $\rho$ is a constant. After integrating out the auxiliary field $A_{0}$, the matrix model action becomes a standard quantum mechanics whose Lagrangian is almost 
the same as that of the soliton approach $[15,16]$,

$$
\begin{aligned}
S=\frac{\lambda N_{c} M_{\mathrm{KK}}}{54 \pi} \int d t \operatorname{tr}_{k}[ & \left(\partial_{0} X^{4}\right)^{2}-\frac{2}{3} M_{\mathrm{KK}}^{2}\left(X^{4}\right)^{2} \\
& \left.\quad+\partial_{0} \bar{w}_{i}^{\dot{\alpha}} \partial_{0} w_{\dot{\alpha} i}-\frac{1}{6} M_{\mathrm{KK}}^{2} \bar{w}_{i}^{\dot{\alpha}} w_{\dot{\alpha} i}-\left(\frac{27 \pi}{\lambda M_{\mathrm{KK}}}\right)^{2} \frac{1}{\bar{w}_{i}^{\dot{\alpha}} w_{\dot{\alpha} i}}\right] .
\end{aligned}
$$

This quantum mechanics is solved by following $[15,16]$. At the leading order in the large $N_{c}$ limit, the wave functions for $X^{4}$ and $\rho$ are classical, which simply means that we can substitute the classical values

$$
X^{4}=0, \quad \rho=2^{-1 / 4} 3^{7 / 4} \sqrt{\pi} \lambda^{-1 / 2} M_{\mathrm{KK}}^{-1} .
$$

The wave functions for the spin/isospin $U$ is nontrivial. They are shared with those of the Skyrme model [11-14], as described in [16], and given by

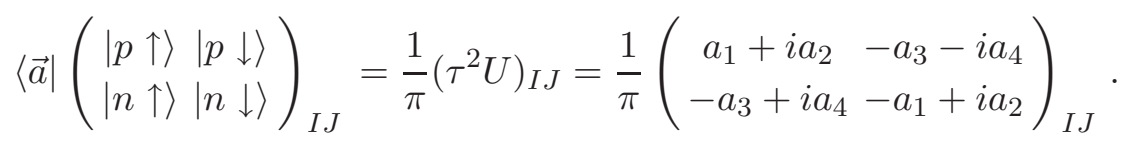

The $\mathrm{SU}(2)$ matrix $U$ is represented by a unit 4 -vector $\vec{a}$ as $U=i a_{i} \tau^{i}+a_{4} \mathbf{1}_{2 \times 2}$, with $\left(a_{1}\right)^{2}+\left(a_{2}\right)^{2}+\left(a_{3}\right)^{2}+\left(a_{4}\right)^{2}=1$.

Next, let us review the case $k=2$ of [1]. The generic solution to the ADHM constraint (2.2) with $k=2, N_{f}=2$ is the well-known ADHM data of two SU(2) YM instantons,

$$
X^{M}=\tau^{3} \frac{r^{M}}{2}+\tau^{1} Y^{M}, \quad w_{\dot{\alpha} i}^{A=1}=U_{\dot{\alpha} i}^{(A=1)} \rho_{1}, \quad w_{\dot{\alpha} i}^{A=2}=U_{\dot{\alpha} i}^{(A=2)} \rho_{2} .
$$

Here the off-diagonal part $Y$ is defined as

$$
Y^{M} \equiv-\frac{\rho_{1} \rho_{2}}{4|r|^{2}} \operatorname{tr}\left[\bar{\sigma}_{M} r^{N} \sigma_{N}\left(\left(U^{(1)}\right)^{\dagger} U^{(2)}-\left(U^{(2)}\right)^{\dagger} U^{(1)}\right)\right],
$$

The vector $r^{M}(M=1,2,3,4)$ is the distance between the two baryons, and $|r|^{2} \equiv\left(r^{M}\right)^{2}$. The $\mathrm{SU}(2)$ matrices $U^{(1)}$ and $U^{(2)}$ denote the moduli parameters of each baryon, while $\rho_{1}$ and $\rho_{2}$ denote the moduli parameter associated with the size of instantons of each baryon. With this choice, the potential associated with $\vec{D}$ (i.e. the ADHM potential) vanishes.

We integrate out the auxiliary field $A_{0}$ to obtain the two-body Hamiltonian. With the $\mathrm{U}(2)$ decomposition $A_{0}=A_{0}^{0} \mathbf{1}_{2 \times 2}+A_{0}^{1} \tau^{1}+A_{0}^{2} \tau^{2}+A_{0}^{3} \tau^{3}$, it is straightforward to evaluate the terms including the gauge field $A_{0}$ in the action,

$$
\begin{aligned}
S_{\text {kin. }+\mathrm{CS}} \equiv \frac{\lambda N_{c} M_{\mathrm{KK}}}{54 \pi} \int d t & \operatorname{tr}\left[\left(D_{0} X^{M}\right)^{2}+D_{0} \bar{w}_{i}^{\dot{\alpha}} D_{0} w_{\dot{\alpha} i}\right]+N_{c} \int d t \operatorname{tr} A_{0} \\
=\frac{\lambda N_{c} M_{\mathrm{KK}}}{54 \pi} \int d t & {\left[2\left(A_{0}^{1}\right)^{2}|r|^{2}+8\left(A_{0}^{3}\right)^{2}\left(Y^{M}\right)^{2}+2\left(\rho_{1}^{2}+\rho_{2}^{2}\right)\left(\left(A_{0}^{0}\right)^{2}+\left(A_{0}^{1}\right)^{2}+\left(A_{0}^{3}\right)^{2}\right)\right.} \\
& \left.\quad+4 \rho_{1} \rho_{2} A_{0}^{0} A_{0}^{1} \operatorname{tr}\left[U^{(1) \dagger} U^{(2)}\right]+4\left(\rho_{1}^{2}-\rho_{2}^{2}\right) A_{0}^{0} A_{0}^{3}+\frac{108 \pi}{\lambda M_{\mathrm{KK}}} A_{0}^{0}\right] \cdot(2.8)
\end{aligned}
$$


Solving the equations of motion for all the components of $A_{0}$ and substituting the solution back to this action, we obtain the potential $\int d t V=-S_{\text {kin.+CS, }}$

$$
\begin{aligned}
V & =2 V_{1-\text { body }}+V_{2-\text { body }}, \quad V_{1-\text { body }}=\frac{27 \pi N_{c}}{4 \lambda M_{\mathrm{KK}}} \frac{1}{\rho^{2}}, \\
V_{2-\text { body }} & =\frac{27 \pi N_{c}}{\lambda M_{\mathrm{KK}}} \frac{\left(u_{0}\right)^{2}}{|r|^{2}+2 \rho^{2}-2\left(u_{0}\right)^{2} \rho^{2}} .
\end{aligned}
$$

Here $u_{0} \equiv(1 / 2) \operatorname{tr}\left[U^{(1) \dagger} U^{(2)}\right]$, and we put $\rho_{1}=\rho_{2}(=\rho)$ which is justified as we keep only the leading term in the large $N_{c}$ expansion. The value of $\rho$ is $(2.4){ }^{1}$

In addition to the terms in $S_{\text {kin.+CS }}$, there is the mass term for $X^{4}$ in the Lagrangian,

$$
\frac{\lambda N_{c} M_{\mathrm{KK}}}{54 \pi} \cdot \frac{2}{3} M_{\mathrm{KK}}^{2} \operatorname{tr}\left(X^{4}\right)^{2}=\frac{\lambda N_{c}}{81 \pi} M_{\mathrm{KK}}^{3}\left(\left(r^{4}\right)^{2} / 2+2\left(Y^{4}\right)^{2}\right) .
$$

The off-diagonal component $Y$ gives an additional two-body potential,

$$
V_{2-\text { body }}^{\text {mass }}=\frac{\lambda N_{c} M_{\mathrm{KK}}^{3}}{162 \pi} \frac{\rho^{4}}{|r|^{4}}\left(r^{i} \operatorname{tr}\left[i \tau^{i}\left(U^{(1)}\right)^{\dagger} U^{(2)}\right]\right)^{2},
$$

where $i=1,2,3$. So the 2-body potential is a sum of (2.10) and (2.13). The fourdimensional distance $|r|$ is equal to the inter-baryon distance $\left|r^{i}\right|^{2}$ in three dimensions, since the classical value of the $X^{4}$ for the single instantons is zero at the large $N_{c}$ leading order, as in (2.4).

Using the nucleon wave function (2.5), it is straightforward to evaluate the vacuum expectation value of this potential. The final form of the two-body nuclear potential is $\langle V\rangle_{I_{1}, J_{1}, I_{2}, J_{2}}=V_{\mathrm{C}}(\vec{r})+S_{12} V_{\mathrm{T}}(\vec{r})$ with the standard definition $S_{12} \equiv 12 J_{1}^{i} \hat{r}^{i} J_{2}^{j} \hat{r}^{j}-4 J_{1}^{i} J_{2}^{i}$ (with $\hat{r}^{i} \equiv r^{i} /|r|, i=1,2,3$ ), where the central and tensor forces are ${ }^{2}$

$$
V_{\mathrm{C}}(\vec{r})=\pi\left(\frac{3^{3}}{2}+8 I_{1}^{i} I_{2}^{i} J_{1}^{j} J_{2}^{j}\right) \frac{N_{c}}{\lambda M_{\mathrm{KK}}} \frac{1}{|r|^{2}}, \quad V_{\mathrm{T}}(\vec{r})=2 \pi I_{1}^{i} I_{2}^{i} \frac{N_{c}}{\lambda M_{\mathrm{KK}}} \frac{1}{|r|^{2}} .
$$

\section{Three-body baryon interaction}

The three-body interaction potential can be computed by using the matrix model with $k=3$ for $k \times k$ matrices. The procedures to compute the nuclear potential are parallel to the case of the two baryons in the previous section, and here is a summary of the procedures:

(1) Choose your $k$, and solve the ADHM constraint (2.2) (which minimizes the potential obtained by integrating out the auxiliary field $D$ ).

\footnotetext{
${ }^{1}$ For our later purpose, we write the expression for the case of classically aligned spins and isospins. This corresponds to $U^{(1)}=U^{(2)}$, which is nothing but an ADHM data for 'tHooft instantons. The two-body potential is found as

$$
V_{2-\text { body }}^{\mathrm{cl}}=\frac{27 \pi N_{c}}{\lambda M_{\mathrm{KK}}} \frac{1}{|r|^{2}}
$$

${ }^{2}$ The result is quite close to that of the soliton approach [17].
} 
(2) Substitute the solution back to the matrix model Lagrangian.

(3) Integrate out the auxiliary field $A_{0}$.

(4) Evaluate the Hamiltonian with your favorite baryon state. The baryon state is just a tensor product of single-baryon states (which is given by the $k=1$ analysis).

In this section, at first as an exercise, we consider a case where all the three baryons share the same classical spin/isospins. Then for next, after giving an explicit set-up for generic quantum spin/isospins, we demonstrate exact computations for baryons aligned on a straight line with equal spacings. The reason for choosing this linear position is just to simplify and illuminate the computations. Finally we evaluate the three-body Hamiltonian with specific three-baryon quantum states: (a) three neutrons with spins averaged, and (b) proton-proton-neutron and proton-neutron-neutron. We find that the three-body nuclear potential is positive i.e. repulsive.

\subsection{An exercise: classical treatment of spin/isospin}

Let us evaluate the three-body Hamiltonian, first, for a simple situation where all the baryons share the same classical spin/isospin, as an exercise.

Procedure (1): solving the ADHM constraint. First, let us consider the configuration space of minimizing the $\vec{D}$ term. This is equivalent to the so-called ADHM constraints (2.2) for any $A, B=1,2,3$. A simple solution to this constraint equation is the ADHM data for 'tHooft instantons, which we treat in this subsection, while in the later subsection we consider generic solution to this constraint. The ADHM data for the 'tHooft instantons consists of diagonal matrices $X$ and special $w$ 's sharing the same orientation,

$$
w_{\dot{\alpha} i}^{A}=U_{\dot{\alpha} i} \rho^{A} \quad(A=1,2,3),
$$

where $2 \times 2$ unitary matrix $U$ is independent of the index $A$. As the degrees of freedom $w$ correspond to the spin and the isospin, this means that all the three baryons share the same "classical" spins and isospins. Here, fixing the orientation $U$ for baryons cannot be achieved with wave functions with finite width, that is the reason we call this "classical." As the off-diagonal elements of $X^{M}$ vanish, all the commutators $[X, X]$ are zero, which trivially satisfies $(2.2)$.

In [8], 'tHooft instantons are used in the soliton approach to evaluate the three-body nuclear forces. The result turns out to vanish. In this subsection, we will find that our matrix model also gives the same answer, the vanishing three-body force for the ADHM data of the 'tHooft instantons.

We are going to choose implicitly the gauge $\partial_{0} w_{\dot{\alpha} i}^{A}=0$ so that there is no timedependence in $w$. See [1] for details of the gauge choices. The matrices $X^{M}$ whose diagonal elements with $M=1,2,3$ specify the spatial location of the baryons are diagonal,

$$
X^{M}=\sum_{a=3,8} \frac{\lambda^{a}}{2} r_{a}^{M}
$$


Procedure (2): substitute the ADHM data to the action. The inter-baryon potential consists of two terms, the potential coming from the integration of $A_{0}$, and the potential from the mass term for $X^{4}$. The latter vanishes for the 'tHooft instantons, as there is no off-diagonal extra component in (3.2). So in this subsection we concentrate on the former.

Given the ADHM data, we can integrate out $A_{0}$, in analogy to the two-body case. The auxiliary field $A_{0}$ is expanded by the Gell-Mann matrices $\lambda^{a}$,

$$
A_{0}=A_{0}^{0} \mathbf{1}_{3 \times 3}+\sum_{a=1}^{8} A_{0}^{a} \frac{\lambda^{a}}{2} .
$$

As in the two-body case, in the Lagrangian the terms containing $A_{0}$ are $\left(D_{0} X\right)^{2}$ and $D_{0} \bar{w} D w$. The CS term contains only the overall U(1) component, $A_{0}^{0}$.

First, the kinetic terms of $X$ gives

$$
\begin{aligned}
\operatorname{tr}\left(D_{0} X^{M}\right)^{2}= & \frac{1}{2}\left(\left(A_{0}^{1} r_{3}^{M}\right)^{2}+\left(A_{0}^{2} r_{3}^{M}\right)^{2}\right)+\frac{1}{8}\left(\left(A_{0}^{4}\right)^{2}+\left(A_{0}^{5}\right)^{2}\right)\left(r_{3}^{M}+\sqrt{3} r_{8}^{M}\right)^{2} \\
& +\frac{1}{8}\left(\left(A_{0}^{6}\right)^{2}+\left(A_{0}^{7}\right)^{2}\right)\left(r_{3}^{M}-\sqrt{3} r_{8}^{M}\right)^{2}
\end{aligned}
$$

Next we consider the kinetic term for $w$,

$$
\operatorname{tr} D_{0} \bar{w}_{i}^{\dot{\alpha}} D_{0} w_{\dot{\alpha} i}=\left(A_{0}^{0}\right)^{2}\left(\sum_{A}\left|w_{\dot{\alpha} i}^{A}\right|^{2}\right)+2 A_{0}^{a} A_{0}^{0} \tilde{j}^{a}+\tilde{t}^{a b} A_{0}^{a} A_{0}^{b}
$$

where $a(=1, \cdots, 8)$ is the adjoint index of the $\mathrm{SU}(3)$, and $A(=1,2,3)$ is the index for the baryons. The coefficients $\tilde{j}^{a}$ and $\tilde{t}^{a b}$ are defined as

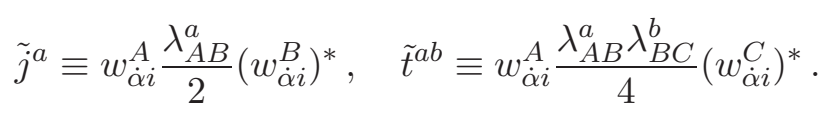

Using the definition of symmetric structure constants $d^{a b c}$ for $\mathrm{SU}(3)$,

$$
\left\{\frac{\lambda^{a}}{2}, \frac{\lambda^{b}}{2}\right\}=d^{a b c} \frac{\lambda^{c}}{2}+\frac{1}{3} \delta^{a b}
$$

the term with $\tilde{t}^{a b}$ is replaced by

$$
\tilde{t}^{a b} A_{0}^{a} A_{0}^{b}=\frac{1}{6} w_{\dot{\alpha} i}^{B}\left(w_{\dot{\alpha} i}^{B}\right)^{*} \delta^{a b} A_{0}^{a} A_{0}^{b}+\frac{d^{a b c}}{4} w_{\dot{\alpha} i}^{A} \lambda_{A C}^{c}\left(w_{\dot{\alpha} i}^{C}\right)^{*} A_{0}^{a} A_{0}^{b} .
$$

Now due to the ADHM data (3.1), all $w_{\dot{\alpha} i}^{A}$ are proportional to each other, i.e., $w_{\dot{\alpha} i}^{A=1} / \rho^{A=1}=$ $w_{\dot{\alpha} i}^{A=2} / \rho^{A=2}=w_{\dot{\alpha} i}^{A=3} / \rho^{A=3}$. Therefore, $w_{\dot{\alpha} i}^{A} \lambda_{A C}^{c}\left(w_{\dot{\alpha} i}^{C}\right)^{*}$ vanishes for $c=2,5,7$, and

$$
\frac{w_{\dot{\alpha} i}^{A} \lambda_{A C}^{c=1}\left(w_{\dot{\alpha} i}^{C}\right)^{*}}{\rho^{1} \rho^{2}}=\frac{w_{\dot{\alpha} i}^{A} \lambda_{A C}^{c=4}\left(w_{\dot{\alpha} i}^{C}\right)^{*}}{\rho^{1} \rho^{3}}=\frac{w_{\dot{\alpha} i}^{A} \lambda_{A C}^{c=6}\left(w_{\dot{\alpha} i}^{C}\right)^{*}}{\rho^{2} \rho^{3}}=2 U_{\dot{\alpha} i}\left(U_{\dot{\alpha} i}\right)^{\dagger}=4 .
$$

Furthermore, similar terms with $c=3$ and $c=8$ are given by

$$
w_{\dot{\alpha} i}^{A} \lambda_{A C}^{c=3}\left(w_{\dot{\alpha} i}^{C}\right)^{*}=2\left(\left(\rho^{1}\right)^{2}-\left(\rho^{2}\right)^{2}\right), \quad w_{\dot{\alpha} i}^{A} \lambda_{A C}^{c=8}\left(w_{\dot{\alpha} i}^{C}\right)^{*}=\frac{2}{\sqrt{3}}\left(\left(\rho^{1}\right)^{2}+\left(\rho^{2}\right)^{2}-2\left(\rho^{3}\right)^{2}\right) .
$$


Using the symmetric structure constant $d^{a b c}$, we get

$$
\begin{aligned}
\tilde{t}^{a b} A_{0}^{a} A_{0}^{b}= & \frac{1}{6} w_{\dot{\alpha} i}^{B}\left(w_{\dot{\alpha} i}^{B}\right)^{*} \delta^{a b} A_{0}^{a} A_{0}^{b}+\frac{d^{a b c}}{4} w_{\dot{\alpha} i}^{A} \lambda_{A C}^{c}\left(w_{\dot{\alpha} i}^{C}\right)^{*} A_{0}^{a} A_{0}^{b} \\
= & \frac{1}{3}\left(\sum_{a=1}^{8}\left(A_{0}^{a}\right)^{2}\right)\left(\left(\rho^{1}\right)^{2}+\left(\rho^{2}\right)^{2}+\left(\rho^{3}\right)^{2}\right)+\left(\frac{2}{\sqrt{3}} A_{0}^{1} A_{0}^{8}+A_{0}^{4} A_{0}^{6}+A_{0}^{5} A_{0}^{7}\right) \rho^{1} \rho^{2} \\
& +\left(-\frac{1}{\sqrt{3}} A_{0}^{4} A_{0}^{8}+A_{0}^{1} A_{0}^{6}-A_{0}^{2} A_{0}^{7}+A_{0}^{3} A_{0}^{4}\right) \rho^{1} \rho^{3} \\
& +\left(-\frac{1}{\sqrt{3}} A_{0}^{6} A_{0}^{8}+A_{0}^{1} A_{0}^{4}+A_{0}^{2} A_{0}^{5}-A_{0}^{3} A_{0}^{6}\right) \rho^{2} \rho^{3} \\
& +\left(\frac{1}{\sqrt{3}} A_{0}^{3} A_{0}^{8}+\frac{1}{4}\left(A_{0}^{4}\right)^{2}+\frac{1}{4}\left(A_{0}^{5}\right)^{2}-\frac{1}{4}\left(A_{0}^{6}\right)^{2}-\frac{1}{4}\left(A_{0}^{7}\right)^{2}\right)\left(\left(\rho^{1}\right)^{2}-\left(\rho^{2}\right)^{2}\right) \\
& +\left(\frac{1}{6}\left(A_{0}^{1}\right)^{2}+\frac{1}{6}\left(A_{0}^{2}\right)^{2}+\frac{1}{6}\left(A_{0}^{3}\right)^{2}-\frac{1}{6}\left(A_{0}^{8}\right)^{2}\right)\left(\left(\rho^{1}\right)^{2}+\left(\rho^{2}\right)^{2}-2\left(\rho^{3}\right)^{2}\right) \\
& +\left(-\frac{1}{12}\left(A_{0}^{4}\right)^{2}-\frac{1}{12}\left(A_{0}^{5}\right)^{2}-\frac{1}{12}\left(A_{0}^{6}\right)^{2}-\frac{1}{12}\left(A_{0}^{7}\right)^{2}\right)\left(\left(\rho^{1}\right)^{2}+\left(\rho^{2}\right)^{2}-2\left(\rho^{3}\right)^{2}\right) .
\end{aligned}
$$

Next, we consider $2 A_{0}^{a} A_{0}^{0} \tilde{j}^{a}$. Again, due to the simplicity of the ADHM data for the 't Hooft instantons, $\tilde{j}^{a}$ is nonzero only for $a=1,4,6,3,8$, and we obtain

$$
\begin{aligned}
2 A_{0}^{a} A_{0}^{0} \tilde{j}^{a}= & 4 A_{0}^{1} A_{0}^{0} \rho^{1} \rho^{2}+4 A_{0}^{4} A_{0}^{0} \rho^{1} \rho^{3}+4 A_{0}^{6} A_{0}^{0} \rho^{2} \rho^{3} \\
& +2 A_{0}^{3} A_{0}^{0}\left(\left(\rho^{1}\right)^{2}-\left(\rho^{2}\right)^{2}\right)+\frac{2}{\sqrt{3}} A_{0}^{8} A_{0}^{0}\left(\left(\rho^{1}\right)^{2}+\left(\rho^{2}\right)^{2}-2\left(\rho^{3}\right)^{2}\right) .
\end{aligned}
$$

In addition, we have $\left(A_{0}^{0}\right)^{2}\left(\sum_{A}\left|w_{\dot{\alpha} i}^{A}\right|^{2}\right)=2\left(\left(\rho^{1}\right)^{2}+\left(\rho^{2}\right)^{2}+\left(\rho^{3}\right)^{2}\right)\left(A_{0}^{0}\right)^{2}$. So, in total, the kinetic term for $w$ is evaluated as

$$
\begin{aligned}
\operatorname{tr} D_{0} \bar{w}_{i}^{\dot{\alpha}} D_{0} w_{\dot{\alpha} i}= & 2\left(\left(\rho^{1}\right)^{2}+\left(\rho^{2}\right)^{2}+\left(\rho^{3}\right)^{2}\right)\left(\left(A_{0}^{0}\right)^{2}+\frac{1}{6} \sum_{a=1}^{8}\left(A_{0}^{a}\right)^{2}\right)+4 \rho^{1} \rho^{2} A_{0}^{1} A_{0}^{0}+4 \rho^{1} \rho^{3} A_{0}^{4} A_{0}^{0} \\
& +4 \rho^{2} \rho^{3} A_{0}^{6} A_{0}^{0}+2 A_{0}^{3} A_{0}^{0}\left(\left(\rho^{1}\right)^{2}-\left(\rho^{2}\right)^{2}\right)+\frac{2}{\sqrt{3}} A_{0}^{8} A_{0}^{0}\left(\left(\rho^{1}\right)^{2}+\left(\rho^{2}\right)^{2}-2\left(\rho^{3}\right)^{2}\right) \\
& +\frac{2 \rho^{1} \rho^{2}}{\sqrt{3}} A_{0}^{1} A_{0}^{8}+\rho^{1} \rho^{2} A_{0}^{4} A_{0}^{6}+\rho^{1} \rho^{2} A_{0}^{5} A_{0}^{7}-\frac{\rho^{1} \rho^{3}}{\sqrt{3}} A_{0}^{4} A_{0}^{8}+\rho^{1} \rho^{3} A_{0}^{1} A_{0}^{6}-\rho^{1} \rho^{3} A_{0}^{2} A_{0}^{7} \\
& +\rho^{1} \rho^{3} A_{0}^{3} A_{0}^{4}-\frac{\rho^{2} \rho^{3}}{\sqrt{3}} A_{0}^{6} A_{0}^{8}+\rho^{2} \rho^{3} A_{0}^{1} A_{0}^{4}+\rho^{2} \rho^{3} A_{0}^{2} A_{0}^{5}-\rho^{2} \rho^{3} A_{0}^{3} A_{0}^{6} \\
& +\left(\frac{1}{\sqrt{3}} A_{0}^{3} A_{0}^{8}+\frac{1}{4}\left(A_{0}^{4}\right)^{2}+\frac{1}{4}\left(A_{0}^{5}\right)^{2}-\frac{1}{4}\left(A_{0}^{6}\right)^{2}-\frac{1}{4}\left(A_{0}^{7}\right)^{2}\right)\left(\left(\rho^{1}\right)^{2}-\left(\rho^{2}\right)^{2}\right) \\
& +\frac{1}{12}\left(2\left(A_{0}^{1}\right)^{2}+2\left(A_{0}^{2}\right)^{2}+2\left(A_{0}^{3}\right)^{2}-2\left(A_{0}^{8}\right)^{2}-\left(A_{0}^{4}\right)^{2}-\left(A_{0}^{5}\right)^{2}-\left(A_{0}^{6}\right)^{2}-\left(A_{0}^{7}\right)^{2}\right) \\
& \times\left(\left(\rho^{1}\right)^{2}+\left(\rho^{2}\right)^{2}-2\left(\rho^{3}\right)^{2}\right) .
\end{aligned}
$$

Finally, the CS term has only the $A_{0}^{0}$ element,

$$
L_{\mathrm{CS}}=\frac{162 \pi}{\lambda M_{K K}} A_{0}^{0} .
$$

The total action $L_{A_{0}}$ involving the gauge field $A_{0}$ is a sum of (3.4), (3.12) and (3.13), as

$$
L_{A_{0}} \equiv \operatorname{tr}\left(D_{0} X^{M}\right)^{2}+\operatorname{tr} D_{0} \bar{w}_{i}^{\dot{\alpha}} D_{0} w_{\dot{\alpha} i}+L_{\mathrm{CS}}
$$


Procedure (3): integrate out $\boldsymbol{A}_{\mathbf{0}}$. We have to solve the simultaneous equations for all $A_{0}^{a}$ and $A_{0}^{0}$,

$$
\frac{\partial L_{A_{0}}}{\partial A_{0}^{0}}=\frac{\partial L_{A_{0}}}{\partial A_{0}^{a}}=0 \quad(\text { for all } a=1, \cdots, 8)
$$

Although $A_{0}^{0}$ is mixed with the other components $A_{0}^{a}$ a unique solution is found as

$$
\begin{aligned}
& A_{0}^{1}=\frac{27 \pi}{\lambda M_{K K}} \frac{\left(\rho^{1}\right)^{2}+\left(\rho^{2}\right)^{2}}{\left(r_{3}^{M}\right)^{2} \rho^{1} \rho^{2}}, \quad A_{0}^{4}=\frac{108 \pi}{\lambda M_{K K}} \frac{\left(\rho^{1}\right)^{2}+\left(\rho^{3}\right)^{2}}{\left(r_{3}^{M}+\sqrt{3} r_{8}^{M}\right)^{2} \rho^{1} \rho^{3}}, \\
& A_{0}^{6}=\frac{108 \pi}{\lambda M_{K K}} \frac{\left(\rho^{2}\right)^{2}+\left(\rho^{3}\right)^{2}}{\left(r_{3}^{M}-\sqrt{3} r_{8}^{M}\right)^{2} \rho^{2} \rho^{3}}, \quad A_{0}^{2}=A_{0}^{5}=A_{0}^{7}=0,
\end{aligned}
$$

$A_{0}^{0}, A_{0}^{3}$ and $A_{0}^{8}$ have complicated expressions, so we omit to write them explicitly here. We plug the solution back to the action $L_{A_{0}}$ given by (3.14), then we obtain integrated action $L_{A_{0}}$ in terms of the moduli parameters $r_{3}^{M}, r_{8}^{M}$ and $\rho$, as

$$
\begin{aligned}
L_{A_{0}}\left(r_{3}^{M}, r_{8}^{M}, \rho^{A}\right)=\left(\frac{54 \pi}{\lambda M_{K K}}\right)^{2}[ & -\sum_{A=1}^{3} \frac{1}{8\left(\rho^{A}\right)^{2}}-\frac{1}{4\left(r_{3}^{M}\right)^{2}}\left(1+\frac{\left(\rho^{1}\right)^{2}}{2\left(\rho^{2}\right)^{2}}+\frac{\left(\rho^{2}\right)^{2}}{2\left(\rho^{1}\right)^{2}}\right) \\
& -\frac{1}{\left(r_{3}^{M}+\sqrt{3} r_{8}^{M}\right)^{2}}\left(1+\frac{\left(\rho^{1}\right)^{2}}{2\left(\rho^{3}\right)^{2}}+\frac{\left(\rho^{3}\right)^{2}}{2\left(\rho^{1}\right)^{2}}\right) \\
& \left.-\frac{1}{\left(r_{3}^{M}-\sqrt{3} r_{8}^{M}\right)^{2}}\left(1+\frac{\left(\rho^{2}\right)^{2}}{2\left(\rho^{3}\right)^{2}}+\frac{\left(\rho^{3}\right)^{2}}{2\left(\rho^{2}\right)^{2}}\right)\right] .
\end{aligned}
$$

The total Hamiltonian (potential) $V^{\mathrm{cl}}$ is given by

$$
S=\frac{\lambda N_{c} M_{K K}}{54 \pi} \int d t L_{A_{0}} \equiv-\int d t V^{\mathrm{cl}}
$$

as in the two-body case. We obtain

$$
\begin{aligned}
V^{\mathrm{cl}}=\left(\frac{54 \pi N_{c}}{\lambda M_{K K}}\right)\left[\sum_{A=1}^{3} \frac{1}{8\left(\rho^{A}\right)^{2}}+\frac{1}{4\left(r_{3}^{M}\right)^{2}}\left(1+\frac{\left(\rho^{1}\right)^{2}}{2\left(\rho^{2}\right)^{2}}+\frac{\left(\rho^{2}\right)^{2}}{2\left(\rho^{1}\right)^{2}}\right)\right. \\
+\frac{1}{\left(r_{3}^{M}+\sqrt{3} r_{8}^{M}\right)^{2}}\left(1+\frac{\left(\rho^{1}\right)^{2}}{2\left(\rho^{3}\right)^{2}}+\frac{\left(\rho^{3}\right)^{2}}{2\left(\rho^{1}\right)^{2}}\right) \\
\left.+\frac{1}{\left(r_{3}^{M}-\sqrt{3} r_{8}^{M}\right)^{2}}\left(1+\frac{\left(\rho^{2}\right)^{2}}{2\left(\rho^{3}\right)^{2}}+\frac{\left(\rho^{3}\right)^{2}}{2\left(\rho^{2}\right)^{2}}\right)\right] .
\end{aligned}
$$

To find the potential intrinsic to the three-body, we need to subtract the one-body and two-body Hamiltonians. For the ADHM data for the 'tHooft instantons, they are given by $[1]$

$$
V_{1-\text { body }}^{\mathrm{cl}}=\frac{27 \pi N_{c}}{4 \lambda M_{\mathrm{KK}}} \frac{1}{\rho^{2}}, \quad V_{2-\text { body }}^{\mathrm{cl}}=\frac{27 \pi N_{c}}{4 \lambda M_{\mathrm{KK}}}\left(2+\frac{\rho_{2}^{2}}{\rho_{1}^{2}}+\frac{\rho_{1}^{2}}{\rho_{2}^{2}}\right) \frac{1}{\left(r^{M}\right)^{2}},
$$


where $r^{M}$ is the distance between the two baryons. The subtraction of these give

$$
V^{\mathrm{cl}}-\sum_{A=1,2,3} V_{1-\text { body }}^{(\mathrm{A}), \mathrm{cl}}-\frac{1}{2} \sum_{A \neq B} V_{2-\text { body }}^{(\mathrm{A}, \mathrm{B}) \mathrm{cl}}=0 .
$$

Therefore, the three-body forces vanish, for the baryons sharing the same classical spin/isospins. This result is the same as the one given in the soliton approach [8].

The "classical" spins and isospins are realized when the magnitude of the spin/isospins is large, which is only possible for heavy higher spin baryons, but not for spin $1 / 2$ nucleons. Therefore unfortunately this "classical" treatment does not work for the realistic nucleons. Next, we keep the quantum spin/isospin degrees of freedom (the phase in $w$ ) explicitly in the computation and provide a framework for nuclear forces with standard quantum spin/isospins.

\subsection{Generic three-body interactions: a set-up}

Procedure (1): solving the ADHM constraint. The computations with the ADHM data for the 'tHooft instantons are easy but they are not realistic system, since the spin/isospin rotation matrix $U$ is fixed by hand. We have to allow arbitrary $U$ for each baryon, in general. This means, instead of the previous (3.1), we allow ${ }^{3}$

$$
w_{\dot{\alpha} i}^{A}=U_{\dot{\alpha} i}^{A} \rho^{A} \quad(A=1,2,3) .
$$

In order to satisfy the ADHM constraint (2.2) with this generic $w$, the off-diagonal components of the matrices $X^{M}$ should be turned on, instead of (3.2),

$$
X^{M}=\sum_{a=3,8} \frac{\lambda^{a}}{2} r_{a}^{M}+\sum_{a=1,4,6} \frac{\lambda^{a}}{2} r_{a}^{M} .
$$

The diagonal $r_{3}$ and $r_{8}$ specify the positions of the three baryons, while the off-diagonal $r_{1}, r_{4}$ and $r_{6}$ are small.

Although generic three-instanton ADHM data is not available, we may need only the ADHM data for well-separated instantons,

$$
\left|r_{3}+\sqrt{3} r_{8}\right| / 2,\left|-r_{3}+\sqrt{3} r_{8}\right| / 2,\left|r_{8}\right| \gg \rho,
$$

since the classical size of the instanton (baryon) is quite small as $\rho \sim 1 / \sqrt{\lambda}$ for large $\lambda$. The ADHM data for the well-separated instantons is described in [18]. In our notation, it

\footnotetext{
${ }^{3}$ Note that in single instanton case, we have gauge freedom from $A_{0}$ to chose this $\partial_{0} w=0$ gauge. In three instanton case, we have $A_{0}^{0}, A_{0}^{3}$, and $A_{0}^{8}$ gauge freedom to choose this $\partial_{0} w^{A=1}=\partial_{0} w^{A=2}=\partial_{0} w^{A=3}=0$.
} 


$$
\begin{aligned}
r_{1}^{M} \sigma_{M}= & \frac{d_{12}^{M} \sigma_{M}}{\left|d_{12}\right|^{2}} \rho_{1} \rho_{2}\left(\left(U^{2}\right)^{\dagger} U^{1}-\left(U^{1}\right)^{\dagger} U^{2}\right) \\
& +\frac{\rho_{1} \rho_{2} \rho_{3}^{2} d_{12}^{M} \sigma_{M}}{4\left|d_{12}\right|^{2}\left|d_{13}\right|^{2}\left|d_{23}\right|^{2}}\left[\left(\left(U^{3}\right)^{\dagger} U^{2}-\left(U^{2}\right)^{\dagger} U^{3}\right) d_{32}^{\dagger} d_{31}\left(\left(U^{1}\right)^{\dagger} U^{3}-\left(U^{3}\right)^{\dagger} U^{1}\right)\right. \\
& \left.-\left(\left(U^{3}\right)^{\dagger} U^{1}-\left(U^{1}\right)^{\dagger} U^{3}\right) d_{31}^{\dagger} d_{32}\left(\left(U^{2}\right)^{\dagger} U^{3}-\left(U^{3}\right)^{\dagger} U^{2}\right)\right]+\mathcal{O}\left(1 / d^{5}\right), \\
r_{4}^{M} \sigma_{M}= & \frac{d_{13}^{M} \sigma_{M}}{\left|d_{13}\right|^{2}} \rho_{1} \rho_{3}\left(\left(U^{3}\right)^{\dagger} U^{1}-\left(U^{1}\right)^{\dagger} U^{3}\right) \\
& +\frac{\rho_{1} \rho_{3} \rho_{2}^{2} d_{13}^{M} \sigma_{M}}{4\left|d_{12}\right|^{2}\left|d_{13}\right|^{2}\left|d_{23}\right|^{2}}\left[\left(\left(U^{2}\right)^{\dagger} U^{3}-\left(U^{3}\right)^{\dagger} U^{2}\right) d_{23}^{\dagger} d_{21}\left(\left(U^{1}\right)^{\dagger} U^{2}-\left(U^{2}\right)^{\dagger} U^{1}\right)\right. \\
& \left.-\left(\left(U^{2}\right)^{\dagger} U^{1}-\left(U^{1}\right)^{\dagger} U^{2}\right) d_{21}^{\dagger} d_{23}\left(\left(U^{3}\right)^{\dagger} U^{2}-\left(U^{2}\right)^{\dagger} U^{3}\right)\right]+\mathcal{O}\left(1 / d^{5}\right) \\
r_{6}^{M} \sigma_{M}= & \frac{d_{23}^{M} \sigma_{M}}{\left|d_{23}\right|^{2}} \rho_{2} \rho_{3}\left(\left(U^{3}\right)^{\dagger} U^{2}-\left(U^{2}\right)^{\dagger} U^{3}\right) \\
& +\frac{\rho_{2} \rho_{3} \rho_{1}^{2} d_{23}^{M} \sigma_{M}}{4\left|d_{12}\right|^{2}\left|d_{13}\right|^{2}\left|d_{23}\right|^{2}}\left[\left(\left(U^{1}\right)^{\dagger} U^{3}-\left(U^{3}\right)^{\dagger} U^{1}\right) d_{13}^{\dagger} d_{12}\left(\left(U^{2}\right)^{\dagger} U^{1}-\left(U^{1}\right)^{\dagger} U^{2}\right)\right. \\
& \left.-\left(\left(U^{1}\right)^{\dagger} U^{2}-\left(U^{2}\right)^{\dagger} U^{1}\right) d_{12}^{\dagger} d_{13}\left(\left(U^{3}\right)^{\dagger} U^{1}-\left(U^{1}\right)^{\dagger} U^{3}\right)\right]+\mathcal{O}\left(1 / d^{5}\right)
\end{aligned}
$$

Here we have defined

$$
d_{i j} \equiv d_{i j}^{M} \sigma_{M}
$$

where $d_{i j}$ is the distance vector between the $i$-th and the $j$-th instantons. From (3.23), the location of the first, second, and third instanton is

$$
r^{M}=r_{3}^{M} / 2+r_{8}^{M} / 2 \sqrt{3},-r_{3}^{M} / 2+r_{8}^{M} / 2 \sqrt{3},-r_{8}^{M} / \sqrt{3},
$$

respectively. Therefore we have

$$
\begin{aligned}
& d_{12}=-d_{21}=r_{3}, \\
& d_{13}=-d_{31}=\left(r_{3}-\sqrt{3} r_{8}\right) / 2, \\
& d_{23}=-d_{32}=\left(-r_{3}-\sqrt{3} r_{8}\right) / 2 .
\end{aligned}
$$

Procedure (2): substitute the ADHM data to the action. As all $U^{A}$ matrices are different, we need to consider $w_{\dot{\alpha} i}^{A} \lambda_{A C}^{c}\left(w_{\dot{\alpha} i}^{C}\right)^{*}$ for all $c=1, \cdots, 8$. However, because $U_{\dot{\alpha} i}^{A} \in$ $\mathrm{SU}(2), w_{\dot{\alpha} i}^{A} \lambda_{A C}^{c}\left(w_{\dot{\alpha} i}^{C}\right)^{*}$ for $c=2,5,7$ vanish as they are proportional to $U_{\dot{\alpha} i}^{A}\left(U_{\dot{\alpha} i}^{B}\right)^{\dagger}-U_{\dot{\alpha} i}^{B}\left(U_{\dot{\alpha} i}^{A}\right)^{\dagger}$

\footnotetext{
${ }^{4}$ Our $r_{1} / 2, r_{4} / 2$ and $r_{6} / 2$ correspond to $b_{12}, b_{13}$, and $b_{23}$ of [18], as they are the off-diagonal elements of the matrix $X^{M}$. Our $\rho_{i} U^{i}$ corresponds to $q_{i}$ of [18]. Our formulas (3.25), (3.26), and (3.26) can be obtained explicitly from eq. (5.13) of [18], by substituting recursively the expression of $b_{i j}$.
} 
with $A, B=1,2,3$. The other components are calculated as follows:

$$
\begin{aligned}
& w_{\dot{\alpha} i}^{A} \lambda_{A C}^{1}\left(w_{\dot{\alpha} i}^{C}\right)^{*}=\rho^{1} \rho^{2}\left(U_{\dot{\alpha} i}^{1}\left(U_{\dot{\alpha} i}^{2}\right)^{\dagger}+U_{\dot{\alpha} i}^{2}\left(U_{\dot{\alpha} i}^{1}\right)^{\dagger}\right)=4 \rho^{1} \rho^{2} u_{0}^{(12)} \\
& w_{\dot{\alpha} i}^{A} \lambda_{A C}^{3}\left(w_{\dot{\alpha} i}^{C}\right)^{*}=2\left(\left(\rho^{1}\right)^{2}-\left(\rho^{2}\right)^{2}\right) \\
& w_{\dot{\alpha} i}^{A} \lambda_{A C}^{4}\left(w_{\dot{\alpha} i}^{C}\right)^{*}=\rho^{1} \rho^{3}\left(U_{\dot{\alpha} i}^{1}\left(U_{\dot{\alpha} i}^{3}\right)^{\dagger}+U_{\dot{\alpha} i}^{3}\left(U_{\dot{\alpha} i}^{1}\right)^{\dagger}\right)=4 \rho^{1} \rho^{3} u_{0}^{(13)} \\
& w_{\dot{\alpha} i}^{A} \lambda_{A C}^{6}\left(w_{\dot{\alpha} i}^{C}\right)^{*}=\rho^{2} \rho^{3}\left(U_{\dot{\alpha} i}^{2}\left(U_{\dot{\alpha} i}^{3}\right)^{\dagger}+U_{\dot{\alpha} i}^{3}\left(U_{\dot{\alpha} i}^{2}\right)^{\dagger}\right)=4 \rho^{2} \rho^{3} u_{0}^{(23)} \\
& w_{\dot{\alpha} i}^{A} \lambda_{A C}^{8}\left(w_{\dot{\alpha} i}^{C}\right)^{*}=\frac{2}{\sqrt{3}}\left(\left(\rho^{1}\right)^{2}+\left(\rho^{2}\right)^{2}-2\left(\rho^{3}\right)^{2}\right) .
\end{aligned}
$$

Here we have used the fact that the $\mathrm{SU}(2)$ matrices $U_{\dot{\alpha} i}^{A}\left(U_{\dot{\alpha} i}^{B}\right)^{\dagger}$ for $A \neq B,(A, B)=1,2,3$ can be written as $u_{0} \mathbf{1}_{2 \times 2}+i \sum_{i=1}^{3} u_{i} \tau^{i}$ with $\sum_{i=0}^{3}\left(u_{i}\right)^{2}=1$, in terms of Pauli matrices $\tau^{i}$, i.e.

$$
U_{\dot{\alpha} i}^{A}\left(U_{\dot{\beta} i}^{B}\right)^{\dagger} \equiv u_{0}^{(A B)}\left(\mathbf{1}_{2 \times 2}\right)_{\dot{\alpha} \dot{\beta}}+i \sum_{i=1}^{3} u_{i}^{(A B)} \tau_{\dot{\alpha} \dot{\beta}}^{i} .
$$

The definition of $u_{0}$ follows that of the two-baryon case, (2.10).

In short, compared with the previous ADHM data for the 'tHooft instantons, we have new parameters $r_{a}^{M}$ with $a=1,4,6$, and $u_{0}^{A B}$. We first describe the integration of $A_{0}$, followed by the explanation of the potential due to the mass term $\operatorname{tr}\left[\left(X^{4}\right)^{2}\right]$.

Let us write the terms including $A_{0}$ in the matrix model action explicitly. They are the kinetic terms of $X$ and $w$, and the CS term. Note that due to the fact that $w_{\dot{\alpha} i}^{A} \lambda_{A C}^{c}\left(w_{\dot{\alpha} i}^{C}\right)^{*}=0$ again for $c=2,5,7$, the calculation for the $w$ kinetic term $D w(D w)^{*}$ is very similar to that of the ADHM data for the 'tHooft instantons. On the other hand, the kinetic term for $X$ contains terms in (3.4) as well as terms including $r_{a}$ with $a=1,4,6$.

$$
\operatorname{tr}\left(D_{0} X^{M}\right)^{2}=\operatorname{tr}\left(-i\left[A_{0}, \sum_{\zeta=1,3,4,6,8} \frac{\lambda^{a}}{2} r_{a}^{M}\right]\right)^{2} .
$$

Due to the fact that terms including $A_{0}^{a}$ with $a=2,5,7$ decouple from the terms including $A_{0}^{b}$ with $b=1,3,4,6,8$, and the fact that all $A_{0}^{a}$ with $a=2,5,7$ appear in the Lagrangian as quadratic terms, the equations of motion for $A_{0}^{a}$ with $a=2,5,7$ are simply solved by $A_{0}^{a}=0(a=2,5,7)$. With this observation, the kinetic term for $X^{M}$ is simplified as

$$
\begin{aligned}
\operatorname{tr}\left(D_{0} X^{M}\right)^{2}= & \frac{1}{8} \\
& \left(\left(A_{0}^{4}\right)^{2} r_{1}^{2}+\left(A_{0}^{6}\right)^{2} r_{1}^{2}+4\left(A_{0}^{1}\right)^{2} r_{3}^{2}+\left(A_{0}^{4}\right)^{2} r_{3}^{2}+\left(A_{0}^{6}\right)^{2} r_{3}^{2}-2 A_{0}^{1} A_{0}^{4} r_{1} r_{4}\right. \\
& -2 \sqrt{3} A_{0}^{6} A_{0}^{8} r_{1} r_{4}+6 A_{0}^{1} A_{0}^{6} r_{3} r_{4}-2 \sqrt{3} A_{0}^{4} A_{0}^{8} r_{3} r_{4}+\left(A_{0}^{1}\right)^{2}\left(r_{4}\right)^{2}+\left(A_{0}^{6}\right)^{2}\left(r_{4}\right)^{2} \\
& +3\left(A_{0}^{8}\right)^{2}\left(r_{4}\right)^{2}-2 A_{0}^{1} A_{0}^{6} r_{1} r_{6}-2 \sqrt{3} A_{0}^{4} A_{0}^{8} r_{1} r_{6}-6 A_{0}^{1} A_{0}^{4} r_{3} r_{6}+2 \sqrt{3} A_{0}^{6} A_{0}^{8} r_{3} r_{6} \\
& -2 A_{0}^{4} A_{0}^{6} r_{4} r_{6}+4 \sqrt{3} A_{0}^{1} A_{0}^{8} r_{4} r_{6} \\
& +\left(A_{0}^{1}\right)^{2}\left(r_{6}\right)^{2}+\left(A_{0}^{4}\right)^{2}\left(r_{6}\right)^{2}+3\left(A_{0}^{8}\right)^{2}\left(r_{6}\right)^{2}+\left(A_{0}^{3}\right)^{2}\left(4\left(r_{1}\right)^{2}+\left(r_{4}\right)^{2}+\left(r_{6}\right)^{2}\right) \\
& +4 \sqrt{3} A_{0}^{4} A_{0}^{6} r_{1} r_{8}+2 \sqrt{3}\left(A_{0}^{4}\right)^{2} r_{3} r_{8}-2 \sqrt{3}\left(A_{0}^{6}\right)^{2} r_{3} r_{8}-2 \sqrt{3} A_{0}^{1} A_{0}^{6} r_{4} r_{8} \\
& -6 A_{0}^{4} A_{0}^{8} r_{4} r_{8}-2 \sqrt{3} A_{0}^{1} A_{0}^{4} r_{6} r_{8}-6 A_{0}^{6} A_{0}^{8} r_{6} r_{8}+3\left(A_{0}^{4}\right)^{2} r_{8}^{2}+3\left(A_{0}^{6}\right)^{2} r_{8}^{2} \\
& -2 A_{0}^{3}\left(4 A_{0}^{1} r_{1} r_{3}+3 A_{0}^{6} r_{1} r_{4}+A_{0}^{4} r_{3} r_{4}-\sqrt{3} A_{0}^{8}\left(r_{4}\right)^{2}\right. \\
& \left.\left.-3 A_{0}^{4} r_{1} r_{6}+A_{0}^{6} r_{3} r_{6}+\sqrt{3} A_{0}^{8}\left(r_{6}\right)^{2}+\sqrt{3} A_{0}^{4} r_{4} r_{8}-\sqrt{3} A_{0}^{6} r_{6} r_{8}\right)\right) .
\end{aligned}
$$


The kinetic term for $w$ is similar to the previous case with the 'tHooft instanton ADHM data.

$$
\begin{aligned}
\operatorname{tr} D_{0} \bar{w}_{i}^{\dot{\alpha}} D_{0} w_{\dot{\alpha} i}= & 2\left(\left(\rho^{1}\right)^{2}+\left(\rho^{2}\right)^{2}+\left(\rho^{3}\right)^{2}\right)\left(\left(A_{0}^{0}\right)^{2}+\frac{1}{6} \sum_{\eta=1,3,4,6,8}\left(A_{0}^{\eta}\right)^{2}\right)+4 \rho^{1} \rho^{2} u_{0}^{(12)} A_{0}^{1} A_{0}^{0} \\
& +4 \rho^{1} \rho^{3} u_{0}^{(13)} A_{0}^{4} A_{0}^{0}+4 \rho^{2} \rho^{3} u_{0}^{(23)} A_{0}^{6} A_{0}^{0}+2 A_{0}^{3} A_{0}^{0}\left(\left(\rho^{1}\right)^{2}-\left(\rho^{2}\right)^{2}\right) \\
& +\frac{2}{\sqrt{3}} A_{0}^{8} A_{0}^{0}\left(\left(\rho^{1}\right)^{2}+\left(\rho^{2}\right)^{2}-2\left(\rho^{3}\right)^{2}\right)+\frac{2 \rho^{1} \rho^{2} u_{0}^{(12)}}{\sqrt{3}} A_{0}^{1} A_{0}^{8}+\rho^{1} \rho^{2} u_{0}^{(12)} A_{0}^{4} A_{0}^{6} \\
& -\frac{\rho^{1} \rho^{3} u_{0}^{(13)}}{\sqrt{3}} A_{0}^{4} A_{0}^{8}+\rho^{1} \rho^{3} u_{0}^{(13)} A_{0}^{1} A_{0}^{6}+\rho^{1} \rho^{3} u_{0}^{(13)} A_{0}^{3} A_{0}^{4} \\
& -\frac{\rho^{2} \rho^{3} u_{0}^{(23)}}{\sqrt{3}} A_{0}^{6} A_{0}^{8}+\rho^{2} \rho^{3} u_{0}^{(23)} A_{0}^{1} A_{0}^{4}-\rho^{2} \rho^{3} u_{0}^{(23)} A_{0}^{3} A_{0}^{6} \\
& +\left(\frac{1}{\sqrt{3}} A_{0}^{3} A_{0}^{8}+\frac{1}{4}\left(A_{0}^{4}\right)^{2}-\frac{1}{4}\left(A_{0}^{6}\right)^{2}\right)\left(\left(\rho^{1}\right)^{2}-\left(\rho^{2}\right)^{2}\right) \\
& +\frac{1}{12}\left(2\left(A_{0}^{1}\right)^{2}+2\left(A_{0}^{3}\right)^{2}-2\left(A_{0}^{8}\right)^{2}-\left(A_{0}^{4}\right)^{2}-\left(A_{0}^{6}\right)^{2}\right)\left(\left(\rho^{1}\right)^{2}+\left(\rho^{2}\right)^{2}-2\left(\rho^{3}\right)^{2}\right) .
\end{aligned}
$$

With the CS term given by (3.13) the total Lagrangian is again written in the form (3.14). Again, we have to solve the simultaneous equations (3.15).

Next, we shall describe the potential due to the mass term of the matrix model,

$$
\begin{aligned}
\frac{\lambda N_{c} M_{\mathrm{KK}}^{3}}{54 \pi} \frac{2}{3} \operatorname{tr}\left[\left(X^{4}\right)^{2}\right]= & \frac{\lambda N_{c} M_{\mathrm{KK}}^{3}}{3^{4} \pi}\left[\frac{1}{4}\left(r_{3}^{4}+r_{8}^{4} / \sqrt{3}\right)^{2}+\frac{1}{4}\left(-r_{3}^{4}+r_{8}^{4} / \sqrt{3}\right)^{2}+\frac{1}{3}\left(r_{8}^{4}\right)^{2}\right. \\
& \left.+\frac{1}{2}\left(\left(r_{1}^{4}\right)^{2}+\left(r_{2}^{4}\right)^{2}+\left(r_{4}^{4}\right)^{2}+\left(r_{5}^{4}\right)^{2}+\left(r_{6}^{4}\right)^{2}+\left(r_{7}^{4}\right)^{2}\right)\right]
\end{aligned}
$$

The first three terms correspond to the square of the diagonal elements $X_{i i}^{4}$ for $i=1,2,3$, so these correspond to the three copies of the one-baryon potential. The terms in the second line are the two-body and the three-body terms. To evaluate these, we need explicit expressions for the off-diagonal $r_{1}, r_{2}, r_{4}, r_{5}, r_{6}$ and $r_{7}$.

In principle, it is a straightforward calculation to determine the three-body force from this. However the actual calculation turns out to be extremely messy, and it is hard to get a physical interpretation from that. To extract the physical essence, next we will choose a particular alignment of the baryons to simplify the expression, so that final answer is easier to analyze.

\subsection{Three-body Hamiltonian for baryons aligned on a line}

We will find that the Hamiltonian is simplified significantly when all the baryons are aligned on a line. We consider the following case

$$
r_{8}^{M}=0, \quad r_{3}^{M} \equiv r^{M} \neq 0 .
$$

This means that the first, the second and the third baryon are placed at $x^{M}=r_{3}^{M} / 2$, $x^{M}=-r_{3}^{M} / 2$, and $x=0$, respectively. In this case, since $r_{8}^{M}=0$, the expression for 
the potential is significantly simplified. Still, since we are treating the spin/isospin moduli quantum mechanically, this gives lots of information on the three-body forces. In addition, we notice that all the size moduli $\rho_{i}$ can be taken to be a classical value, $\rho_{1}=\rho_{2}=\rho_{3}=\rho$, since we are dealing with only the leading order in the large $N_{c}$ limit. The large $N_{c}$ limit is the same as classical limit since the action (2.1) has overall $N_{c}$. This simplifies the computation too.

The resultant Lagrangian concerning the gauge field $A_{0}$ is

$$
L_{A_{0}}=\frac{\lambda M_{\mathrm{KK}} N_{c}}{54 \pi}\left(L_{1}+L_{2}\right)
$$

where

$$
\begin{aligned}
L_{1} \equiv & \frac{162 A_{0}^{0} \pi}{\lambda M_{\mathrm{KK}}}+\frac{\left(A_{0}^{1}\right)^{2} r^{2}}{2}+\frac{\left(A_{0}^{4}\right)^{2} r^{2}}{8}+\frac{\left(A_{0}^{6}\right)^{2} r^{2}}{8} \\
& +\left(6\left(A_{0}^{0}\right)^{2}+\left(A_{0}^{1}\right)^{2}+\left(A_{0}^{3}\right)^{2}+\left(A_{0}^{4}\right)^{2}+\left(A_{0}^{6}\right)^{2}+\left(A_{0}^{8}\right)^{2}\right) \rho^{2} \\
& +\left(A_{0}^{4} A_{0}^{6}+\frac{2 A_{0}^{1} A_{0}^{8}}{\sqrt{3}}\right) \rho^{2} u_{0}^{(12)}+\left(A_{0}^{3} A_{0}^{4}+A_{0}^{1} A_{0}^{6}-\frac{A_{0}^{4} A_{0}^{8}}{\sqrt{3}}\right) \rho^{2} u_{0}^{(13)} \\
& +\left(A_{0}^{1} A_{0}^{4}-A_{0}^{3} A_{0}^{6}-\frac{A_{0}^{6} A_{0}^{8}}{\sqrt{3}}\right) \rho^{2} u_{0}^{(23)}+4 A_{0}^{0}\left(A_{0}^{1} u_{0}^{(12)}+A_{0}^{4} u_{0}^{(13)}+A_{0}^{6} u_{0}^{(23)}\right) \rho^{2}, \\
4 L_{2} \equiv & 2\left(A_{0}^{3}\right)^{2}\left(r_{1}^{M}\right)^{2}+\frac{1}{2}\left(A_{0}^{4}\right)^{2}\left(r_{1}^{M}\right)^{2}+\frac{1}{2}\left(A_{0}^{6}\right)^{2}\left(r_{1}^{M}\right)^{2}-A_{0}^{1} A_{0}^{4} r_{1}^{M} r_{4}^{M}-3 A_{0}^{3} A_{0}^{6} r_{1}^{M} r_{4}^{M} \\
& -\sqrt{3} A_{0}^{6} A_{0}^{8} r_{1}^{M} r_{4}^{M}+\frac{1}{2}\left(A_{0}^{1}\right)^{2}\left(r_{4}^{M}\right)^{2}+\frac{1}{2}\left(A_{0}^{3}\right)^{2}\left(r_{4}^{M}\right)^{2}+\frac{1}{2}\left(A_{0}^{6}\right)^{2}\left(r_{4}^{M}\right)^{2}+\sqrt{3} A_{0}^{3} A_{0}^{8}\left(r_{4}^{M}\right)^{2} \\
& +\frac{3}{2}\left(A_{0}^{8}\right)^{2}\left(r_{4}^{M}\right)^{2}+3 A_{0}^{3} A_{0}^{4} r_{1}^{M} r_{6}^{M}-A_{0}^{1} A_{0}^{6} r_{1}^{M} r_{6}^{M}-\sqrt{3} A_{0}^{4} A_{0}^{8} r_{1}^{M} r_{6}^{M}-A_{0}^{4} A_{0}^{6} r_{4}^{M} r_{6}^{M} \\
& +2 \sqrt{3} A_{0}^{1} A_{0}^{8} r_{4}^{M} r_{6}^{M}+\frac{1}{2}\left(A_{0}^{1}\right)^{2}\left(r_{6}^{M}\right)^{2}+\frac{1}{2}\left(A_{0}^{3}\right)^{2}\left(r_{6}^{M}\right)^{2}+\frac{1}{2}\left(A_{0}^{4}\right)^{2}\left(r_{6}^{M}\right)^{2} \\
& -\sqrt{3} A_{0}^{3} A_{0}^{8}\left(r_{6}^{M}\right)^{2}+\frac{3}{2}\left(A_{0}^{8}\right)^{2}\left(r_{6}^{M}\right)^{2} .
\end{aligned}
$$

For getting this expression of $L_{A_{0}}=L_{1}+L_{2}$, we have used the equations

$$
r_{3}^{M} r_{1}^{M}=0, \quad\left(r_{3}^{M}+\sqrt{3} r_{8}^{M}\right) r_{4}^{M}=0, \quad\left(r_{3}^{M}-\sqrt{3} r_{8}^{M}\right) r_{6}^{M}=0,
$$

to eliminate cross terms between $r_{3,8}$ and $y$ in the Lagrangian. These can be shown explicitly using the solution of the ADHM constraint (2.2) in the expansion of the small $\rho^{2} / r^{2}$. The expansion was studied in detail in [18]. Using the expression given in eq. (5.13) of [18], it is easy to show the equations above. This elimination of the cross terms is important for a simplification of the computations. In fact, we can show later that $L_{2}$ is not necessary, when integrating out $A_{0}$.

Next we evaluate the contribution from the mass term $\left(X^{4}\right)^{2}$. The alignment (3.39) simplifies the ADHM data (3.25), (3.26) and (3.27) quite a lot. In fact, we have

$$
\begin{aligned}
& r_{1}^{M} \sigma_{M}=\frac{1}{|r|^{2}} \rho_{1} \rho_{2} r T_{21}-\frac{1}{|r|^{4}} \rho_{1} \rho_{2} \rho_{3}^{2} r\left(T_{32} T_{13}-T_{13} T_{32}\right)+\mathcal{O}\left(1 /|r|^{5}\right), \\
& r_{4}^{M} \sigma_{M}=\frac{2}{|r|^{2}} \rho_{1} \rho_{3} r T_{31}-\frac{1}{|r|^{4}} \rho_{1} \rho_{3} \rho_{2}^{2} r\left(T_{32} T_{12}-T_{12} T_{32}\right)+\mathcal{O}\left(1 /|r|^{5}\right), \\
& r_{6}^{M} \sigma_{M}=-\frac{2}{|r|^{2}} \rho_{2} \rho_{3} r T_{32}+\frac{1}{|r|^{4}} \rho_{2} \rho_{3} \rho_{1}^{2} r\left(T_{31} T_{21}-T_{21} T_{31}\right)+\mathcal{O}\left(1 /|r|^{5}\right),
\end{aligned}
$$


where $r \equiv r^{M} \sigma_{M}$, and $T_{i j} \equiv\left(U^{i}\right)^{\dagger} U^{j}-\left(U^{j}\right)^{\dagger} U^{i}=-T_{j i}$. Note that the first term in each of the right hand side equals the off-diagonal entry of the two-body case, $Y$ in (2.7). The second terms are corrections due to the three-body effect. So, the three-body contribution in the mass term $\operatorname{tr}\left(X^{4}\right)^{2}$ (3.38) should appear at the leading order as a liner term in these second terms, multiplied by the first terms. An explicit computation leads to

$$
\begin{aligned}
V_{3-\text { body }}^{\text {mass }}= & \frac{\lambda N_{c} M_{\mathrm{KK}}^{3}}{2^{2} 3^{4} \pi} \frac{\rho^{6}}{|r|^{6}} \\
& \times\left(\operatorname{tr}\left[r T_{21}\right] \operatorname{tr}\left[r\left(T_{23} T_{13}-T_{13} T_{23}\right)\right]-2 \operatorname{tr}\left[r T_{31}\right] \operatorname{tr}\left[r\left(T_{32} T_{12}-T_{12} T_{32}\right)\right]\right. \\
& \left.\quad-2 \operatorname{tr}\left[r T_{32}\right] \operatorname{tr}\left[r\left(T_{31} T_{21}-T_{21} T_{31}\right)\right]\right) .
\end{aligned}
$$

We have already subtracted the one-body and the two-body potentials here, and took $\rho_{1}=\rho_{2}=\rho_{3}=\rho$ which is the classical value (the leading value in the large $N_{c}$ expansion).

Procedure (3): integrating out $\boldsymbol{A}_{\mathbf{0}}$. Once we solve the simultaneous equations of motion for (3.15) for $A_{0}^{\zeta}$, and plug the solutions into the Lagrangian $L_{A_{0}}$, we should obtain

$$
L_{A_{0}}=-V, \quad V \equiv \sum_{A=1,2,3} V_{1-\text { body }}^{(A)}+\frac{1}{2} \sum_{A \neq B} V_{2-\text { body }}^{(A, B)}+V_{3-\text { body }}
$$

where the first term is the one-body rest energy, and the second term is the two-body interaction potential. As obtained in [1], their expressions are

$$
V_{1-\text { body }}^{(A)}=\frac{27 \pi N_{c}}{4 \lambda M_{\mathrm{KK}}} \frac{1}{\rho^{2}}, \quad V_{2-\text { body }}^{(A, B)}=\frac{27 \pi N_{c}}{\lambda M_{\mathrm{KK}}} \frac{\left(u_{0}^{(A B)}\right)^{2}}{\left|r^{(A B)}\right|^{2}+2 \rho^{2}-2\left(u_{0}^{(A B)}\right)^{2} \rho^{2}} .
$$

Here the inter-nucleon distance is, according to our alignment (3.39),

$$
\left|r^{(12)}\right|=r, \quad\left|r^{(13)}\right|=\left|r^{(23)}\right|=r / 2 .
$$

The third term $V_{3 \text {-body }}$ is what we like to compute.

We are interested in the regime of short distances $r \ll 1 / M_{\mathrm{KK}}$. However, as the classical size of the baryon $\rho$ is quite small, $\rho \sim 1 /\left(\sqrt{\lambda} M_{\mathrm{KK}}\right)$, the region of our interest is rather a "long-distance" expansion $\rho \ll r$ in effect. Therefore we need to expand the resultant Hamiltonian for small $\rho / r$. As we look at the Lagrangian $L_{A_{0}}=L_{1}+L_{2}$, we notice that $L_{2}$ is of order $\rho^{4} / r^{2}$, as we know that the ADHM constraint is solved in this expansion as $y=\mathcal{O}\left(\rho^{2} / r\right)$. On the other hand, As is obvious from (3.47), the two-body interaction is $\mathcal{O}\left(1 / r^{2}\right)$ so the three-body interaction should start from $\rho^{2} / r^{4}$. (This is suggested also from the soliton approach, see [8].) Therefore, $L_{2}$ is not necessary as it is at higher order in this expansion. ${ }^{5}$

The Lagrangian $L_{1}$ can be conveniently written as

$$
L_{1}=\vec{A}^{T} M \vec{A}+\vec{B}^{T} \vec{A}
$$

\footnotetext{
${ }^{5}$ As a check, we can perform a computation with keeping $L_{2}$ explicitly to confirm this. The computation is lengthy and is not presented in this manuscript, but we have confirmed it. Note that for the generic case with nonzero $r_{8}$, this simplification is not expected, because in general there are terms of the form $r_{8} y$ which contributes additionally to $L_{1}$, so one needs explicit expression for $y$ by solving the ADHM constraints.
} 
where

$$
\begin{aligned}
\vec{A}^{T} & \equiv\left(A_{0}^{0}, A_{0}^{1}, A_{0}^{3}, A_{0}^{4}, A_{0}^{6}, A_{0}^{8}\right), \quad \vec{B}^{T} \equiv \frac{162 \pi}{\lambda M_{\mathrm{KK}}}(1,0,0,0,0,0), \\
M & \equiv P+Q, \quad P \equiv \frac{r^{2}}{8} \operatorname{diag}(0,4,0,1,1,0) \\
Q & \equiv \rho^{2}\left(\begin{array}{cccccc}
6 & 2 u_{0}^{(12)} & 0 & 2 u_{0}^{(13)} & 2 u_{0}^{(23)} & 0 \\
2 u_{0}^{(12)} & 1 & 0 & u_{0}^{(23)} / 2 & u_{0}^{(13)} / 2 & u_{0}^{(12)} / \sqrt{3} \\
0 & 0 & 1 & u_{0}^{(13)} / 2 & -u_{0}^{(23)} / 2 & 0 \\
2 u_{0}^{(13)} & u_{0}^{(23)} / 2 & u_{0}^{(13)} / 2 & 1 & u_{0}^{(12)} / 2 & -u_{0}^{(13)} / 2 \sqrt{3} \\
2 u_{0}^{(23)} & u_{0}^{(13)} / 2 & -u_{0}^{(23)} / 2 & u_{0}^{(12)} / 2 & 1 & -u_{0}^{(23)} / 2 \sqrt{3} \\
0 & u_{0}^{(12)} / \sqrt{3} & 0 & -u_{0}^{(13)} / 2 \sqrt{3} & -u_{0}^{(23)} / 2 \sqrt{3} & 1
\end{array}\right) .
\end{aligned}
$$

Since $M$ is a symmetric matrix i.e. $M^{T}=M$, the equations of motion for $A_{0}$ is solved by

$$
\vec{A}=-\frac{1}{2} M^{-1} \vec{B}
$$

which is substituted back to $L_{1}$ to give the Hamiltonian (which is $-L_{A_{0}}$ )

$$
V=\frac{\lambda M_{\mathrm{KK}} N_{c}}{54 \pi} \cdot \frac{1}{4} \vec{B}^{T} M^{-1} \vec{B} .
$$

As $\vec{B}$ has only one non-zero entry, this is nothing but

$$
V=\frac{3^{5} \pi N_{c}}{2 \lambda M_{\mathrm{KK}}}\left[M^{-1}\right]_{(1,1)}
$$

which can be evaluated using the first cofactor of the matrix $M$. By expanding in power series of $\rho^{2} / r^{2}$ up to $O\left(\rho^{4} / r^{6}\right)$, we obtain,

$$
V=\frac{3^{5} \pi N_{c}}{2 \lambda M_{\mathrm{KK}}}\left(\frac{1}{6 \rho^{2}}+\frac{2\left(u^{(1,2)}\right)^{2}+8\left(u^{(1,3)}\right)^{2}+8\left(u^{(2,3)}\right)^{2}}{9 r^{2}}+\frac{4 \rho^{2} f_{\mathrm{SI}}}{9 r^{4}}\right)+\mathcal{O}\left(\rho^{4} / r^{6}\right),
$$

where spin/isospin phase $f_{\mathrm{SI}}$ is defined as

$$
\begin{aligned}
f_{\mathrm{SI}} \equiv & \left(u_{0}^{(1,2)}\right)^{4}-\left(u_{0}^{(1,2)}\right)^{2}+16\left(u_{0}^{(1,3)}\right)^{4}-16\left(u_{0}^{(1,3)}\right)^{2}+16\left(u_{0}^{(2,3)}\right)^{4}-16\left(u_{0}^{(2,3)}\right)^{2} \\
& +4\left(u_{0}^{(1,2)}\right)^{2}\left(u_{0}^{(1,3)}\right)^{2}+4\left(u_{0}^{(1,2)}\right)^{2}\left(u_{0}^{(2,2)}\right)^{2}+16\left(u_{0}^{(1,3)}\right)^{2}\left(u_{0}^{(2,3)}\right)^{2} \\
& -24 u_{0}^{(1,2)} u_{0}^{(2,3)} u_{0}^{(1,3)}
\end{aligned}
$$

Subtracting the 1-body and 2-body potentials (3.48) from this expression as in (3.47), we obtain the potential intrinsic to the three-body nature by the expansion of $\rho^{2} / r^{2}$, which we call $V_{3-\text { body }}^{A_{0}}$ as

$$
\begin{aligned}
V_{3-\text { body }}^{A_{0}}=\frac{216 \pi N_{c} \rho^{2}}{\lambda M_{\mathrm{KK}}|r|^{4}}[ & \left(u_{0}^{(1,2)}\right)^{2}\left(u_{0}^{(1,3)}\right)^{2}+\left(u_{0}^{(1,2)}\right)^{2}\left(u_{0}^{(2,3)}\right)^{2}+4\left(u_{0}^{(1,3)}\right)^{2}\left(u_{0}^{(2,3)}\right)^{2} \\
& \left.-6 u_{0}^{(1,2)} u_{0}^{(2,3)} u_{0}^{(1,3)}\right]+\mathcal{O}\left(\rho^{4} / r^{6}\right) .
\end{aligned}
$$


With the potential coming from the $X^{4}$ mass term (3.46), the total three-body potential is

$$
V_{3-\text { body }}=V_{3-\text { body }}^{A_{0}}+V_{3-\text { body }}^{\text {mass }}
$$

With this at hand, we can evaluate this potential with any three-baryon state with any spin/isospin. Next, we shall choose two wave functions, one is appropriate for the neutron stars, and the other is for a Helium-3 nucleus and a triton (a nucleus of tritium), to find the three-body nuclear potential is positive.

We have two remarks on (3.59). First, the three-body Hamiltonian (3.59) is of order $\mathcal{O}\left(1 /\left(\lambda^{2} r^{4}\right)\right)$ because of $\rho \sim 1 / \sqrt{\lambda}$, and so it is suppressed by $1 / \lambda^{2}$. This is consistent with the generic observation given in the soliton picture [8] stating that the generic $k$-body potential is of order $1 /\left(\lambda^{k-1} r^{2 k-2}\right)$ in the unit of $M_{\mathrm{KK}}=1$. Second, in the expression above if we put all the matrices $U^{(i)}$ equal to each other so that the ADHM data is that of the 'tHooft instantons, we have $u_{0}^{(i, j)}=1$ and $A_{i j}=0$, resulting in the vanishing three-body potential. This is consistent with the result of the previous section.

Procedure (4): evaluate the Hamiltonian with baryon states. Now, we are ready to compute the spin/isospin dependence of the three-body nuclear force at short distances. Although we can evaluate it for any choice of spin/isospin for each baryon, in this paper we choose the following two states as explicit examples:

(4-a) three neutrons with spins averaged.

(4-b) proton-proton-neutron (and proton-neutron-neutron).

The reason for these choices is that the first example is relevant for dense states of many neutrons such as core of neutron stars and supernovae, where the three-body nuclear forces are quite important. The second is obviously for the spectrum of Helium-3 nucleus where three-body forces are expected to contribute, and also for a triton.

(4-a): three neutrons with spins averaged. For protons and neutrons, the singlebaryon wave function is given by (2.5). For the neutron stars and the supernovae, we need neutron states with spins averaged. Thus, for any given operator of the quantum mechanics, the appropriate expectation value for these is obtained by

$$
\langle V\rangle=\frac{1}{2}[\langle n \uparrow|\widehat{\mathcal{O}}| n \uparrow\rangle+\langle n \downarrow|\widehat{\mathcal{O}}| n \downarrow\rangle]
$$

For the case of $\widehat{\mathcal{O}}$ being the three-body Hamiltonian, we need to take the above expectation value for each of three baryons. As nucleons are fermions, any wave functions should be anti-symmetric under the exchange of the nucleons. Here, as three neutrons move around in realistic situations, we do not anti-symmetrize the wave functions ${ }^{6}$ (in this paper we have not evaluated nuclear potentials coming from motion of the baryons).

\footnotetext{
${ }^{6}$ In fact, once we take three neutrons for the isospin sector, it is impossible to anti-symmetrize the wave function with the spin sector, without a help of angular momenta.
} 
Here for a demonstration, let us consider a single baryon case (3.61). Using the coordinate expression of the wave functions, this (3.61) means

$$
\langle V\rangle=\int d \Omega_{3} \frac{1}{2}\left[\mathcal{O}|\langle\vec{a} \mid n \uparrow\rangle|^{2}+\mathcal{O}|\langle\vec{a} \mid n \downarrow\rangle|^{2}\right]
$$

Here $d \Omega_{3}$ is the integration over the $S^{3}$ spanned by the unit vector $\vec{a}$. Using the wave functions (2.5), we can see

$$
|\langle\vec{a} \mid n \uparrow\rangle|^{2}+|\langle\vec{a} \mid n \downarrow\rangle|^{2}=\frac{1}{\pi^{2}}\left[\left(a_{1}\right)^{2}+\left(a_{2}\right)^{2}+\left(a_{3}\right)^{2}+\left(a_{4}\right)^{2}\right]=\frac{1}{\pi^{2}}
$$

So, we obtain a simple expression

$$
\langle V\rangle=\frac{1}{2 \pi^{2}} \int d \Omega_{3} \mathcal{O} .
$$

Using this simple formula, the three-body potential with the spin-averaged wave function is

$$
\begin{aligned}
& \left\langle V_{3-\text { body }}^{A_{0}}\right\rangle_{\text {nnn(spin-averaged })} \\
& =\frac{216 \pi N_{c} \rho^{2}}{\lambda M_{\mathrm{KK}}|r|^{4}} \frac{1}{\left(2 \pi^{2}\right)^{3}} \int d \Omega_{3}^{(1)} d \Omega_{3}^{(2)} d \Omega_{3}^{(3)}\left[\left(u_{0}^{(1,2)}\right)^{2}\left(u_{0}^{(1,3)}\right)^{2}+\left(u_{0}^{(1,2)}\right)^{2}\left(u_{0}^{(2,3)}\right)^{2}\right. \\
& \left.+4\left(u_{0}^{(1,3)}\right)^{2}\left(u_{0}^{(2,3)}\right)^{2}-6 u_{0}^{(1,2)} u_{0}^{(2,3)} u_{0}^{(1,3)}\right] .
\end{aligned}
$$

This integral over three $S^{3}$ 's can be easily performed. For example, for $\left(u_{0}^{(1,2)}\right)^{2}$, using the definition below (2.5) and (2.10), we get

$$
u_{0}^{(i, j)}=\frac{1}{2} \operatorname{tr}\left[U^{(i) \dagger} U^{(j)}\right]=\vec{a}^{(i)} \cdot \vec{a}^{(j)},
$$

where $\vec{a}^{(i)}$ is unit 4-component vector, pointing one phase point on $S^{3}$ for spin/isospin d.o.f. $U^{(i)}$. Therefore, we obtain

$$
\int d \Omega_{3}^{(1)}\left(u_{0}^{(1,2)}\right)^{2}=\int d \Omega_{3}^{(1)} \cos ^{2} \theta=\int \cos ^{2} \theta \sin ^{2} \theta \sin \tilde{\theta} d \theta d \tilde{\theta} d \tilde{\tilde{\theta}}=\frac{\pi^{2}}{2},
$$

where $\theta$ is the angle between $\vec{a}^{(1)}$ and $\vec{a}^{(2)}$. Using this and also the following integral

$$
\int d \Omega_{3}^{(1)} d \Omega_{3}^{(2)} d \Omega_{3}^{(3)} u_{0}^{(1,2)} u_{0}^{(2,3)} u_{0}^{(1,3)}=\frac{\pi^{6}}{2}
$$

we obtain

$$
\left\langle V_{3-\text { body }}^{A_{0}}\right\rangle_{\text {nnn(spin-averaged })}=0 .
$$

Therefore the three-body potential from the $A_{0}$ term vanishes for the spin-averaged neutron wave function. 
In the same manner, for $V_{3-\text { body }}^{\text {mass }}$, the expectation value is given as

$$
\begin{aligned}
\left\langle V_{3 \text {-body }}^{\text {mass }}\right. & \rangle_{\text {nnn(spin-averaged })}=\frac{\lambda N_{c} M_{\mathrm{KK}}^{3}}{2^{2} 3^{4} \pi} \frac{\rho^{6}}{|r|^{6}} \\
& \times \frac{1}{\left(2 \pi^{2}\right)^{3}} \int d \Omega_{3}^{(1)} d \Omega_{3}^{(2)} d \Omega_{3}^{(3)}\left(\operatorname{tr}\left[r T_{21}\right] \operatorname{tr}\left[r\left(T_{23} T_{13}-T_{13} T_{23}\right)\right]\right. \\
& \left.-2 \operatorname{tr}\left[r T_{31}\right] \operatorname{tr}\left[r\left(T_{32} T_{12}-T_{12} T_{32}\right)\right]-2 \operatorname{tr}\left[r T_{32}\right] \operatorname{tr}\left[r\left(T_{31} T_{21}-T_{21} T_{31}\right)\right]\right) \\
= & -\frac{\lambda N_{c} M_{\mathrm{KK}}^{3}}{2^{2} 3^{3} \pi\left(2 \pi^{2}\right)^{3}} \frac{\rho^{6}}{|r|^{6}} \int d \Omega_{3}^{(1)} d \Omega_{3}^{(2)} d \Omega_{3}^{(3)}\left(\operatorname{tr}\left[r T_{21}\right] \operatorname{tr}\left[r\left(T_{23} T_{13}-T_{13} T_{23}\right)\right]\right) .
\end{aligned}
$$

Here in the last equality we have used the invariance under the exchange of the integration variables, $d \Omega_{3}^{(1)} \leftrightarrow d \Omega_{3}^{(2)} \leftrightarrow d \Omega_{3}^{(3)}$. The integration can be performed by using the polar coordinates of the $S^{3}$, and the result is

$$
\frac{1}{\left(2 \pi^{2}\right)^{3}} \int d \Omega_{3}^{(1)} d \Omega_{3}^{(2)} d \Omega_{3}^{(3)}\left(\operatorname{tr}\left[r T_{21}\right] \operatorname{tr}\left[r\left(T_{23} T_{13}-T_{13} T_{23}\right)\right]\right)=-8|\vec{r}|^{2},
$$

where $\vec{r}=\left(r^{1}, r^{2}, r^{3}\right)$ is the three-dimensional vector which specifies the inter-baryon distance in our space. At the leading order in $1 / N$ expansion, we may use the classical value for $r^{4}$ which is zero, so in effect the three-dimensional distance is equal to the four-dimensional one, $|\vec{r}|=|r|$. We can substituting the classical value $\rho=2^{-1 / 4} 3^{7 / 4} \sqrt{\pi} \lambda^{-1 / 2} M_{\mathrm{KK}}^{-1}$ at the leading order in the $1 / N_{c}$ expansion. So, we obtain the three-body potential due to the matrix model mass term as

$$
\left\langle V_{3-\text { body }}^{\text {mass }}\right\rangle_{\text {nnn(spin-averaged })}=\frac{2^{-1 / 2} 3^{15 / 2} \pi^{2} N_{c}}{\lambda^{2} M_{\mathrm{KK}}^{3}|r|^{4}} .
$$

Therefore, in total, we obtain

$$
\begin{aligned}
\left\langle V_{3-\text { body }}\right\rangle_{\text {nnn(spin-averaged })} & =\left\langle V_{3-\text { body }}^{A_{0}}\right\rangle_{\text {nnn(spin-averaged })}+\left\langle V_{3-\text { body }}^{\text {mass }}\right\rangle_{\text {nnn(spin-averaged })} \\
& =\frac{2^{-1 / 2} 3^{15 / 2} \pi^{2} N_{c}}{\lambda^{2} M_{\mathrm{KK}}^{3}|r|^{4}}
\end{aligned}
$$

This is the three-body nuclear potential for three neutrons placed on a line with equal spacings $|r| / 2$, with spins averaged. The three-body potential is suppressed compared to the two-body potential by $\sim 1 / \lambda\left(r M_{K K}\right)^{2} \ll 1$ for large $\lambda$, which is generic hierarchy between $N+1$-body potential to $N$-body one as shown in [8]. $M_{\mathrm{KK}}$ roughly indicates the QCD scale, and our computation is valid at short-distance, $1 /\left(\sqrt{\lambda} M_{\mathrm{KK}}\right) \ll|r| \ll 1 / M_{\mathrm{KK}} \cdot{ }^{7}$

(4-b): proton-proton-neutron. Let us evaluate the three-body potential with the case of proton-proton-neutron. We are interested in the three-nucleon state with a total spin $1 / 2$ and a total isospin $1 / 2$. For any choice of the third component of the spin/isospins, we can find a unique wave function with a complete anti-symmetrization.

\footnotetext{
${ }^{7}$ This $M_{\mathrm{KK}}$ is about $1 \mathrm{GeV}$ if it is fit with $\rho$ meson mass [5], while it is about $0.5 \mathrm{GeV}$ when it is fit with baryon mass differences [16]. We are working in the large $\lambda$ expansion. The 'tHooft coupling constant of $\mathrm{QCD}, \lambda$, is $\mathcal{O}(10-20)$ when it is fit with pion decay constant [5].
} 
The proton-proton-nuetron means the third component of the total isospin is $+1 / 2$. For example, when the third component of the total spin is $+1 / 2$,

$$
\begin{aligned}
& \frac{1}{\sqrt{6}}\left[|p \uparrow\rangle_{1}|p \downarrow\rangle_{2}|n \uparrow\rangle_{3}-|p \downarrow\rangle_{1}|p \uparrow\rangle_{2}|n \uparrow\rangle_{3}-|p \uparrow\rangle_{1}|n \uparrow\rangle_{2}|p \downarrow\rangle_{3}\right. \\
& \left.\quad+|p \downarrow\rangle_{1}|n \uparrow\rangle_{2}|p \uparrow\rangle_{3}-|n \uparrow\rangle_{1}|p \downarrow\rangle_{2}|p \uparrow\rangle_{3}+|n \uparrow\rangle_{1}|p \uparrow\rangle_{2}|p \downarrow\rangle_{3}\right] .
\end{aligned}
$$

The calculation with this wave function is straightforward, and we find the integrals

$$
\begin{aligned}
\int d \Omega_{3}^{(1)} d \Omega_{3}^{(2)} d \Omega_{3}^{(3)}\left|\psi\left(\vec{a}_{1}, \vec{a}_{2}, \vec{a}_{3}\right)\right|^{2}\left(u_{0}^{(1,2)}\right)^{2}\left(u_{0}^{(1,3)}\right)^{2} & =\frac{1}{36} \\
\int d \Omega_{3}^{(1)} d \Omega_{3}^{(2)} d \Omega_{3}^{(3)}\left|\psi\left(\vec{a}_{1}, \vec{a}_{2}, \vec{a}_{3}\right)\right|^{2} u_{0}^{(1,2)} u_{0}^{(2,3)} u_{0}^{(1,3)} & =\frac{1}{36} \\
\int d \Omega_{3}^{(1)} d \Omega_{3}^{(2)} d \Omega_{3}^{(3)}\left|\psi\left(\vec{a}_{1}, \vec{a}_{2}, \vec{a}_{3}\right)\right|^{2}\left(\operatorname{tr}\left[r T_{21}\right] \operatorname{tr}\left[r\left(T_{23} T_{13}-T_{13} T_{23}\right)\right]\right) & =-\frac{320}{27}|\vec{r}|^{2} .
\end{aligned}
$$

Using these formula, we obtain again

$$
\left\langle V_{3-\text { body }}^{A_{0}}\right\rangle_{\mathrm{ppn}}=0
$$

while for the other potential we have a different factor

$$
\left\langle V_{3-\text { body }}^{\text {mass }}\right\rangle_{\mathrm{ppn}}=\frac{2^{5 / 2} 3^{9 / 2} 5 \pi^{2} N_{c}}{\lambda^{2} M_{\mathrm{KK}}^{3}|r|^{4}} .
$$

Therefore, in total, the three-body potential is

$$
\left\langle V_{3-\text { body }}\right\rangle_{\mathrm{ppn}}=\left\langle V_{3-\text { body }}^{A_{0}}\right\rangle_{\mathrm{ppn}}+\left\langle V_{3-\text { body }}^{\text {mass }}\right\rangle_{\mathrm{ppn}}=\frac{2^{5 / 2} 3^{9 / 2} 5 \pi^{2} N_{c}}{\lambda^{2} M_{\mathrm{KK}}^{3}|r|^{4}} .
$$

The three-body potential is positive, that means, we have a repulsive three-body force at short distances.

This computation is for $(+1 / 2,+1 / 2)$ of the third components of the spin and the isospin. Computations with three other wave functions, $(+1 / 2,-1 / 2),(-1 / 2,+1 / 2)$, and $(-1 / 2,-1 / 2)$, can be done in the same manner, and the result for the three-body potential turns out to be the same as (3.78) for all of these. These are due to the fact that the action (2.1) has rotational invariance $\mathrm{SO}(3)$ and isospin $\mathrm{SU}(2)$ invariance. This includes the case for proton-neutron-neutron, which is the case for a triton (a tritium nucleus).

\section{Summary and discussions}

With the simple $\mathrm{U}(k)$ matrix model for $k$-nucleon systems which we proposed in [1] together with $\mathrm{P}$. Yi, in this paper we have computed short-distance three-body nuclear forces. Our matrix model is not a phenomenological model, but derived in string theory using the gauge/string duality (the AdS/CFT correspondence). More precisely, our matrix model is a low-energy effective field theory on baryon vertex D4-branes [7] in the D4-D8 holographic model $[5,6]$ of large $N_{c}$ QCD. In this framework, we can compute nuclear potentials for arbitrary number $k$ of the nucleons. 
Our computations are straightforward. For three nucleons, we took $k=3$, i.e. U(3) matrix model. The matrix model Hamiltonian evaluated with a quantum three-baryon state, a tensor product of single-baryon states, gives the three-body nuclear potential. We subtracted one-body and two-body contributions, thus the remaining is the force intrinsic to the three-body. The computations are valid only at short range, $1 / \sqrt{\lambda} M_{\mathrm{KK}} \ll|r| \ll$ $1 / M_{\mathrm{KK}}$ where $\lambda$ is the 'tHooft coupling constant of the QCD which is $\mathcal{O}(10)$ for fitting pion decay constant [5], and $M_{\mathrm{KK}}$ is $\mathcal{O}(1-0.5) \mathrm{GeV}[5,16]$ when it is fit with meson/baryon masses (or mass differences). As explicit examples, we took a) three neutrons with spins averaged, and b) proton-proton-neutron, both aligned on a line with equal spacings. The resultant three-body nuclear potentials are (3.73) and (3.78), both of which are positive.

Let us discuss possible importance of our result. We have computed (3.73) for threebody neutrons. But as seen from the form of wave functions (2.5) and isospin $\mathrm{SU}(2)$ invariance of the action (2.1), the results hold also for three-body forces for three-protons. Therefore the result (3.73) hold as far as all three nucleons have same flavor. In the same manner, the three-body potential (3.78) for the proton-proton-neutron is equal to the threebody potential for proton-neutron-neutron, which is responsible for a triton. These results imply that there are additional repulsive forces in addition to two-body forces for these states at short distances.

The three-body potentials which we obtained in (3.73) and (3.78) are suppressed by $1 / \lambda\left(r M_{K K}\right)^{2}$ compared with two-body potential, and at the length scale where our computation is valid, i.e. $1 /\left(\sqrt{\lambda} M_{\mathrm{KK}}\right) \ll|r| \ll 1 / M_{\mathrm{KK}}$, this suppression factor $1 / \lambda\left(r M_{K K}\right)^{2}$ is small. This makes our three-body potential computation valid; we have two-body dominant repulsive potential and furthermore small but nonzero repulsive potential from three-body forces.

These three-body forces are stronger as distances get shorter. As a result, at very short distances where neutrons are highly dense, three-body forces give additional repulsive forces. This statement supports recent observation that the nuclear two-body repulsion is not enough to explain supernovae explosions, nor the equations of states for the core of neutron stars. In high density nuclear matters such as the neutron stars, our result suggests that the repulsive core of neutrons in neutron stars and supernovae has an extra positive contribution besides the repulsive potential from the two-body nuclear potential. The necessity of the repulsive three-body forces for neutrons has been indicated by analysis of mass bounds of neutron stars and supernova explosion simulations.

We also found that the three-body forces for proton-proton-neutron at short distances is repulsive. In Helium-3 nuclear spectrum, it is expected that a short-range repulsive threebody forces is necessary, and our result sounds to be consistent with this. Furthermore, we found a repulsive three-body forces also for a proton-neutron-neutron, which should be related to a triton. There are other related issues in few-body nuclear spectra. ${ }^{8}$

Our example is limited to three nucleons on a line, so this is not conclusive for the questions concerning the interesting situations listed above. However, our results are suggestive. In principle, it is very straightforward to compute the $k$-body forces at arbitrary

\footnotetext{
${ }^{8}$ Spectra of heavy nuclei have been discussed in a holographic approach in [19, 20].
} 
arrangements of nucleons using our matrix model, therefore this matrix model is effective for studying short-range many-body nuclear forces.

\section{Acknowledgments}

We would like to thank P. Yi for collaboration on the earlier project [1]. We would also like to thank S. Aoki, T. Doi, T. Hatsuda, T. Nakatsukasa, K. Sekiguchi, and G. Watanabe for helpful discussions and comments. K.H. and N.I. would like to thank Yukawa Institute for Theoretical Physics. K.H. thanks CERN. N.I. also thanks RIKEN. This research was partially supported by KAKENHI Grant-in-Aid 21105514, 19740125, 22340069.

Open Access. This article is distributed under the terms of the Creative Commons Attribution Noncommercial License which permits any noncommercial use, distribution, and reproduction in any medium, provided the original author(s) and source are credited.

\section{References}

[1] K. Hashimoto, N. Iizuka and P. Yi, A Matrix Model for Baryons and Nuclear Forces, JHEP 10 (2010) 003 [arXiv: 1003.4988] [SPIRES].

[2] J.M. Maldacena, The large- $N$ limit of superconformal field theories and supergravity, Adv. Theor. Math. Phys. 2 (1998) 231 [Int. J. Theor. Phys. 38 (1999) 1113] [hep-th/9711200] [SPIRES].

[3] S.S. Gubser, I.R. Klebanov and A.M. Polyakov, Gauge theory correlators from non-critical string theory, Phys. Lett. B 428 (1998) 105 [hep-th/9802109] [SPIRES].

[4] E. Witten, Anti-de Sitter space and holography, Adv. Theor. Math. Phys. 2 (1998) 253 [hep-th/9802150] [SPIRES].

[5] T. Sakai and S. Sugimoto, Low energy hadron physics in holographic QCD, Prog. Theor. Phys. 113 (2005) 843 [hep-th/0412141] [SPIRES].

[6] T. Sakai and S. Sugimoto, More on a holographic dual of QCD, Prog. Theor. Phys. 114 (2005) 1083 [hep-th/0507073] [SPIRES].

[7] E. Witten, Baryons and branes in anti de Sitter space, JHEP 07 (1998) 006 [hep-th/9805112] [SPIRES].

[8] K. Hashimoto, N. Iizuka and T. Nakatsukasa, N-Body Nuclear Forces at Short Distances in Holographic QCD, Phys. Rev. D 81 (2010) 106003 [arXiv:0911.1035] [SPIRES].

[9] N. Dorey, T.J. Hollowood, V.V. Khoze and M.P. Mattis, The calculus of many instantons, Phys. Rept. 371 (2002) 231 [hep-th/0206063] [SPIRES].

[10] M.F. Atiyah, N.J. Hitchin, V.G. Drinfeld and Y.I. Manin, Construction of instantons, Phys. Lett. A 65 (1978) 185 [SPIRES].

[11] T.H.R. Skyrme, A Unified Field Theory Of Mesons And Baryons, Nucl. Phys. 31 (1962) 556 [SPIRES].

[12] T.H.R. Skyrme, A Nonlinear field theory, Proc. Roy. Soc. Lond. A 260 (1961) 127 [SPIRES].

[13] T.H.R. Skyrme, Particle states of a quantized meson field, Proc. Roy. Soc. Lond. A 262 (1961) 237 [SPIRES]. 
[14] G.S. Adkins, C.R. Nappi and E. Witten, Static Properties of Nucleons in the Skyrme Model, Nucl. Phys. B 228 (1983) 552 [SPIRES].

[15] D.K. Hong, M. Rho, H.-U. Yee and P. Yi, Chiral dynamics of baryons from string theory, Phys. Rev. D 76 (2007) 061901 [hep-th/0701276] [SPIRES].

[16] H. Hata, T. Sakai, S. Sugimoto and S. Yamato, Baryons from instantons in holographic QCD, Prog. Theor. Phys. 117 (2007) 1157 [hep-th/0701280] [SPIRES].

[17] K. Hashimoto, T. Sakai and S. Sugimoto, Nuclear Force from String Theory, Prog. Theor. Phys. 122 (2009) 427 [arXiv:0901.4449] [SPIRES].

[18] N.H. Christ, E.J. Weinberg and N.K. Stanton, General self-dual Yang-Mills solutions, Phys. Rev. D 18 (1978) 2013 [SPIRES].

[19] K. Hashimoto, Holographic Nuclei, Prog. Theor. Phys. 121 (2009) 241 [arXiv:0809.3141] [SPIRES].

[20] K. Hashimoto, Holographic Nuclei : Supersymmetric Examples, JHEP 12 (2009) 065 [arXiv:0910.2303] [SPIRES]. 